\title{
Depth of cohomology support loci for quasi-projective varieties via orbifold pencils
}

\author{
Enrique Artal Bartolo, José I. Cogolludo-Agustín \\ and Anatoly Libgober
}

\begin{abstract}
We describe several relations between a homological invariant of characters of fundamental groups of projective manifolds called depth and maps onto orbicurves. This extends previously studied relations between families of local systems and holomorphic maps onto hyperbolic curves. First, we derive the existence of characters whose depth is bounded below by the number of independent orbifold pencils. Conversely, for some class of characters, we deduce the existence of as many independent pencils as the depth of the character. Second, we show a new relation between depth of characters of the fundamental group and solutions of a certain Diophantine equation (related to the Pell equation) over the field of rational functions. Finally we give a Hodge theoretical characterization of essential coordinate characters of the fundamental groups of the complements to plane curves, i.e., characters whose existence cannot be detected by considering the homology of branched abelian covers.
\end{abstract}

\section{Introduction}

Understanding the fundamental groups of the complements to plane algebraic curves is an old problem. Though a procedure for their calculation, due to Zariski [38] and van Kampen [23], is well known and has been implemented in many examples, it sheds little light on the possible structure, properties, or geometric significance of the fundamental groups.

Already Zariski [37] discovered that the noncommutativity of the fundamental group of the complement of a curve with nodes and cusps is related to the position of the cusps. Since then a precise relation has been found between the fundamental group and the dimensions of linear systems of plane curves associated with arbitrary singularities (see [24]). This provided a geometric viewpoint on funda-

Mathematics Subject Classification (2010): 14H30, 14J30, 14H50, 11G05, 57M12, $14 \mathrm{H} 52$.

Keywords: Algebraic curves, characteristic varieties, Albanese maps, orbifold pencils, Pell's equation on function fields. 
mental groups though further development has been hindered by the difficulty of the problems about the positions of the singularities of curves.

A new approach emerged more recently albeit in the case of reducible curves (see [3], [26]). The noncommutativity of $\pi_{1}\left(\mathbb{P}^{2} \backslash C\right)$ arose as a consequence of the existence of holomorphic maps of $\mathbb{P}^{2} \backslash C$ onto hyperbolic curves (see [3]). Such maps correspond to pencils of curves in $\mathbb{P}^{2}$ such that $C$ contains a union of a finite number of its members. The existence of such pencils is related to the invariants of the quotient of the fundamental group of $\mathbb{P}^{2} \backslash C$ by its second commutator (see [26]). These invariants are subvarieties of the torus of characters of the fundamental group called the characteristic varieties (see Section 2.1) that extend the classical notion of Alexander polynomial (whose roots are also elements of the torus $\mathbb{C}^{*}$ ).

In this approach the components of characteristic varieties having dimension greater than one correspond to holomorphic maps from $\mathbb{P}^{2} \backslash C$ onto hyperbolic curves. One-dimensional components were shown to be related to maps onto $\mathbb{C}^{*}$ (see [16], [7]). Further work showed that one-dimensional components (and some zero-dimensional components as well) can be detected by showing the existence of maps of the complements $\mathbb{P}^{2} \backslash C$ onto hyperbolic or elliptic orbifolds (see [7]). Such maps correspond to orbifold pencils related to $C$, i.e., to a curve in $\mathbb{P}^{2}$ such that a finite number of its members are unions of irreducible components of $C$ and extra components with multiplicity.

The present paper provides new conditions for the existence of orbifold pencils and new relations between pencils and fundamental groups providing methods to detect their noncommutativity (in fact to obtain much more precise information).

One of the main results of the paper, Theorem 1.3, gives a lower bound for the dimension of the twisted cohomology in degree one of the local system associated with the character of a quasi-projective group in terms of the number of independent orbifold pencils. As a partial converse, in the case of 2-torsion characters, positive depth implies the existence of orbifold pencils with base $\mathbb{C}_{2,2}$. This extends previous results by the second and third authors (see [11]).

The other two main results are in the context of plane curve complements. Theorem 1.4 characterizes an interesting class of characters (called essential coordinate characters) in terms of the Hodge structure of the complement. Theorem 1.5 states that the set of orbifold pencils mentioned above admits a group structure. In fact it resembles that of the solutions to the Pell equation $u^{2}-f(x) v^{2}=1$ over the function field $\mathbb{C}(x)$ (see Section 5.2). Hence the existence of orbifold pencils with nontrivial orbifold structure is equivalent to the existence of solutions for Diophantine equations of the form $f(x, y) u^{2}-g(x, y) v^{2}=h(x, y)$ over the function field $\mathbb{C}(x, y)$, providing new connections between such equations and fundamental groups.

These main results have been used in [5] to give a detailed description of some of the most archetypical examples of plane curves whose fundamental group of the complement admits essential coordinate characters of order 2. Quasitoric relations, Pell equations, orbifold pencils, and their independence are dealt with in more detail. 
To illustrate this arithmetic relation consider the type $B_{3}$ reflection arrangement, which can be given as the union of the following nine lines in $\mathbb{P}^{2}$ :

(CEVA)

$$
\begin{array}{lll}
\ell_{1}:=x, & \ell_{2}:=y, & \ell_{3}:=z, \\
\ell_{4}:=(y-z), & \ell_{5}:=(x-z), & \ell_{6}:=(x-y), \\
\ell_{7}:=(x-y-z), & \ell_{8}:=(y-z-x), & \ell_{9}:=(z-x-y) .
\end{array}
$$

Note that these lines satisfy the equations

$$
\begin{aligned}
& \ell_{2} \ell_{5}-\ell_{3} \ell_{6}=\ell_{1} \ell_{4}, \\
& \ell_{2} \ell_{6}-\ell_{3} \ell_{5}=\ell_{4} \ell_{7},
\end{aligned}
$$

and

$$
\ell_{2} \ell_{5} \ell_{8}^{2}-\ell_{3} \ell_{6} \ell_{9}^{2}=\ell_{1} \ell_{4} \ell_{7}^{2}
$$

(relation (P3) was noted in [29] and later, independently, in [17]). Let $D$ stand for the so-called Ceva-type arrangement, i.e., the union of the seven lines $\left\{\ell_{1}, \ldots, \ell_{7}\right\}$. One can rephrase relation (P1) geometrically as the existence of a holomorphic map from the complement of the set of solutions to $\ell_{2} \ell_{5}=\ell_{3} \ell_{6}=\ell_{1} \ell_{4}=0$ onto $\mathbb{P}^{1}$ for which $\left\{\ell_{2} \ell_{5}=0\right\},\left\{\ell_{3} \ell_{6}=0\right\}$, and $\left\{\ell_{1} \ell_{4}=0\right\}$ are the preimages of three different points of $\mathbb{P}^{1}$ (and similarly for relation (P2)). On the other hand, relation (P3) shows the existence of a holomorphic dominant map $\mathbb{P}^{2} \backslash D \rightarrow \mathbb{C}_{2,2}$ for which $\left\{\ell_{8}=0\right\}$, and $\left\{\ell_{9}=0\right\}$ have multiplicity 2 and are the preimages of the two orbifold points. Later we will refer to such a map as an orbifold pencil (see Section 4.2 for detailed definitions). This easily shows the existence of different surjections of $\pi_{1}\left(\mathbb{P}^{2} \backslash D\right)$ onto free groups of rank 2 (for (P1) and (P2)) and the metacyclic group $\mathbb{Z}_{2} * \mathbb{Z}_{2}$ (for $(\mathrm{P} 3)$ ). Other interesting insights into the Cevatype arrangement and the deleted $B_{3}$-arrangement $\left\{\ell_{1}, \ldots, \ell_{8}\right\}$ can be found in the conceptual sequel of this paper by the same authors devoted to expanding this and other examples (see [5]).

The paper's structure is outlined now. The first part of Section 1 is devoted to describing background material on the relation between fundamental groups and ordinary pencils as well as previous results on the relation between the Alexander polynomials of plane curves with nodes and cusps and orbifold pencils. These results are essential for the second part of this section, where the main results of the paper are stated and briefly discussed. In Sections 2 and 3 we give an account of recent developments about the variety of characters of quasiprojective fundamental groups and its connection with Alexander polynomials and Albanese varieties of cyclic covers. Section 4.1 introduces the main properties of orbifold pencils and contains a proof of Theorem 1.3. Section 5 focuses on the case of hypersurface complements in $\mathbb{P}^{n}$, or equivalently curve complements in $\mathbb{P}^{2}$. In the first part a proof of Theorem 1.4, that is, a Hodge-theoretical characterization of essential coordinate characters, is given, and in the second part the arithmetical connection between such characters and the equations of the curve are described. Finally 
we devote Section 6 to briefly analyzing two typical examples of plane curves for which the fundamental groups admit order-2 characters. The first serves to stress that the Hodge-theoretical condition on the weight of the character is essential for the existence of orbifold pencil maps. The second example, also introduced above, shows how the existence of essential coordinate characters of order 2 is associated with the group structure of the quasitoric relations of their equations, also described as solutions to a Pell equation. This last section is revisited in the sequel to this paper in [5].

\section{Background and statements of results}

Let $\mathcal{X}$ be a smooth quasi-projective variety and let $\chi \in \operatorname{Hom}\left(\pi_{1}(\mathcal{X}), \mathbb{C}^{*}\right)$ be a character of its fundamental group. Viewing $\chi$ as a rank one local system, one associates to it the twisted cohomology groups. The purpose of this note is to extend known relations between holomorphic maps of $\mathcal{X}$ onto curves, i.e., holomorphic pencils, and dimensions of the twisted cohomology $H^{1}(\mathcal{X}, \chi)$.

The problem of the existence of holomorphic pencils can be traced back almost one hundred years and in its projective version (where local systems are replaced by holomorphic bundles) goes back to Castelnuovo, deFranchis, Catanese, GreenLazarsfeld, and Simpson (see [27] for an extense list of references). The quasiprojective case was considered in [3], where the structure of the jumping subsets of the character variety

$$
\stackrel{\circ}{V}_{k}(\mathcal{X}):=\left\{\chi \in \operatorname{Hom}\left(\pi_{1}(\mathcal{X}), \mathbb{C}^{*}\right) \mid \operatorname{dim} H^{1}(\mathcal{X}, \chi)=k\right\}
$$

was studied together with its relation to pencils. In this context, if $\chi \in \stackrel{\circ}{V}_{k}(\mathcal{X})$ we say $\chi$ has depth $k$. The characteristic varieties $V_{k}(\mathcal{X})$ are defined analogously to $\stackrel{\circ}{V}_{k}(\mathcal{X})$, but replacing $=$ by $\geq$ in (1.1). This term was introduced in [26] for complements to plane curves and explicitly related to the structure of the fundamental group in [21], [26]. To be more precise, the characteristic varieties referred to above can be described as the zero sets of the Fitting ideals of the abelianization $\pi_{1}^{\prime} / \pi_{1}^{\prime \prime}$ of the commutator of $\pi_{1}$, which coincide with the jumping loci (1.1) outside of the trivial character (see Theorem 2.3). In particular, the characteristic varieties (unlike the jumping sets for the higher cohomology spaces) depend only on the fundamental group. Fox calculus provides an effective method for calculating the characteristic varieties in the cases when a presentation of the fundamental group by generators and relators is known.

The results of [3] are as follows. Each $V_{k}(\mathcal{X}) \subset \operatorname{Hom}\left(\pi_{1}(\mathcal{X}), \mathbb{C}^{*}\right)$ is a finite union of translated subgroups (i.e., cosets) of $\operatorname{Hom}\left(\pi_{1}(\mathcal{X}), \mathbb{C}^{*}\right)$. Moreover, for each component of positive dimension there exists a curve $C$ with negative Euler characteristic such that this component has the form $\rho \cdot f^{*} \operatorname{Hom}\left(\pi_{1}(C), \mathbb{C}^{*}\right)$ for some holomorphic map $f: \mathcal{X} \rightarrow C$. This was supplemented in [27] by showing that the zero-dimensional components have finite order.

A more precise version of this result can be found in [7] in terms of orbifolds. It includes some missing points regarding resonance conditions and extends the result from $V_{1}(\mathcal{X})$ to all characteristic varieties $V_{k}(\mathcal{X}), k \geq 1$. 
If $\mathcal{X}$ is a complement to a plane projective curve, the target $C$ of a holomorphic pencil mentioned above must be necessarily $C=\mathbb{P}^{1} \backslash\{$ points $\}$ and thus $f$ extends to a rational pencil on $\mathbb{P}^{2}$. In this case, positive-dimensional translated components $\rho \cdot f^{*} \operatorname{Hom}\left(\pi_{1}(C), \mathbb{C}^{*}\right)$ of $V_{1}(C)$ have been shown (see Dimca [16]) to be related to the multiple fibers of such a pencil; see also [7].

For a generic nonisolated character $\chi \in V_{k}(C)$ in a component of $V_{k}(C)$ of dimension greater than one, the depth is given by the formula

$$
\operatorname{dim} H^{1}(C, \chi)= \begin{cases}\operatorname{dim} V_{k}(C)-2=-e(C) & \text { if } C \text { is compact } \\ \operatorname{dim} V_{k}(C)-1=-e(C) & \text { otherwise }\end{cases}
$$

where $e(C)$ is the (topological) Euler characteristic of $C$. For example, if $C=$ $\mathbb{P}^{1} \backslash\{n$ points $\}$, then $\operatorname{dim} H^{1}\left(C, \mathbb{C}^{*}\right)=n-1$ and $\operatorname{dim} H^{1}(C, \chi)=-e(C)=n-2$, if $\chi$ is nontrivial.

This provides a simple way to determine or at least to estimate the depth of characters on components having positive dimension.

Isolated points in components $V_{k}(\mathcal{X})$ are a common occurrence and below we describe the geometric significance of the depth of zero-dimensional irreducible components of $V_{k}(\mathcal{X})$. We do so using orbifold pencils associated with such characters (as was mentioned, such characters must have finite order).

It is worth mentioning that the nature of the cohomology of the local systems is essentially different depending on whether $H_{1}(\overline{\mathcal{X}}, \mathbb{C})$ is trivial or not. In the latter case, due to the surjection $\pi_{1}(\mathcal{X}) \rightarrow \pi_{1}(\overline{\mathcal{X}}) \rightarrow 1$, some of the characters of $\pi_{1}(\mathcal{X})$ are the characters of the projective fundamental group (see [35] for a discussion on the difference between the projective and the quasi-projective case). In this paper (as in [3]) we focus on the case when $H_{1}(\overline{\mathcal{X}}, \mathbb{C})=0$. This includes the case of the complements to plane curves which provides many concrete and interesting examples.

For the basics on the theory of orbifolds we refer to [2] or, since we shall consider mainly orbifold curves, to [32] or [18]. An orbifold pencil is a (birational) dominant map $\mathcal{X} \rightarrow \mathcal{C}$, where $\mathcal{C}$ is the orbicurve such that the preimage of each point in $\mathcal{C}$ with stabilizer of order $m$ is a multiple fiber of order a multiple of $m$. A proof of Lemma 1.1 can be found in [7].

Lemma 1.1. An orbifold pencil $f: \mathcal{X} \rightarrow \mathcal{C}$ defines a morphism of orbifold fundamental groups $f_{*}: \pi_{1}(\mathcal{X}) \rightarrow \pi_{1}^{\text {orb }}(\mathcal{C})$.

We call (see Definition 4.8) the map given in Lemma 1.1 a marked orbifold pencil $f: \mathcal{X} \rightarrow \mathcal{C}$. The markings are given by the pairs $(\mathcal{X}, \chi), \chi \in V_{k}(\mathcal{X})$, and $(\mathcal{C}, \rho), \rho \in V_{k}^{\text {orb }}(\mathcal{C})$, such that $f^{*}(\rho)=\chi$, where $f^{*}$ is the map of groups of characters corresponding to $f_{*}$. Note that $V_{k}^{\text {orb }}(\mathcal{C})$ is the orbifold characteristic variety of $\mathcal{C}$ defined as $V_{k}$ in (1.1) for $\pi_{1}^{\text {orb }}(\mathcal{C})$, which only depends on the group, as mentioned above.

A pair $(\mathcal{X}, \chi)$ can be marked by several orbifold pencils and we show that the number of such markings is related in an appropriate sense to the depth (see Theorem 1.2 below and Section 4.3). 
The relation between orbifold pencils and local systems with nonvanishing cohomology was studied in [11]. In that paper, the problem of finding a bound on the degree of the Alexander polynomial $\Delta(t)$ is discussed for plane curves with cusps and nodes as the only singularities (or curves with singularities in a more general class of $\delta$-essential singularities). The connection with the cohomology of local systems comes from the following: for an irreducible curve $D$ in $\mathbb{P}^{2}$ one has $H_{1}\left(\mathbb{P}^{2} \backslash D, \mathbb{Z}\right)=\mathbb{Z} / \operatorname{deg} D \mathbb{Z}$, i.e., $\operatorname{Hom}\left(\pi_{1}(\mathcal{X}), \mathbb{C}^{*}\right)=\mu_{\operatorname{deg} D}$ (the group of $\operatorname{deg} D$-roots of unity in $\left.\mathbb{C}^{*}\right)$. If $\chi_{\xi}$ corresponds to $\xi \in \mu_{\operatorname{deg} D}$ then

$$
\Delta(\xi)=0 \Longrightarrow \operatorname{dim} H^{1}\left(\mathbb{P}^{2} \backslash D, \chi_{\xi}\right) \neq 0 .
$$

The key step in [11] for obtaining the bound on the degree of the Alexander polynomial (or equivalently the multiplicity of the root $\exp (2 \pi i / 6)$ ) was to show the following theorem.

Theorem 1.2. The degree of the Alexander polynomial of a curve D having cusps and nodes as its only singularities coincides with the number of independent orbifold pencils $\mathbb{P}^{2} \rightarrow \mathbb{P}_{2,3,6}^{1}$ such that $D$ is the preimage of the orbifold point having the cyclic group of order six as its stabilizer. This number of independent pencils equals the rank of the group of quasitoric relations

$$
u^{2}+v^{3}=w^{6} F
$$

where $F=0$ is a defining equation for $D$.

Theorem 1.2 can also be extended to general Alexander polynomials and nonreduced curves.

One of the main results of this paper is the following theorem (proved in Section 4.3) providing the relations between orbifold pencils and depth. It shows that the number of independent pencils (with a given target) provides a lower bound for the depth of a character. Moreover, for an interesting class of characters this bound is exact.

Theorem 1.3. Let $\mathcal{X}$ be a quasi-projective manifold together with a character $\chi$.

(1) Assume that there are $n$ strongly independent marked orbifold pencils on $(\mathcal{X}, \chi)$ with a fixed target $(\mathcal{C}, \rho)$ and let $d(\rho)$ denote the depth of the character $\rho$ of $\pi_{1}^{\text {orb }}(\mathcal{C})$. Then $d(\chi) \geq n d(\rho)$.

(2) If, in addition, $\chi$ is a 2-torsion character and 2 is its only weight (see 2.9 for a definition of weights of a character), then there are exactly $d(\chi)$ strongly independent orbifold pencils on $\mathcal{X}$ whose target is the global $\mathbb{Z}_{2}$-orbifold $\mathcal{C}=$ $\mathbb{C}_{2,2}$. These pencils are marked with the nontrivial character $\rho$ of $\pi_{1}^{\text {orb }}\left(\mathbb{C}_{2,2}\right)$ characterized by the condition that it extends to $\mathbb{P}_{2,2}^{1}$.

Moreover, if $\chi$ is a d-torsion character, then (1) implies that $\phi_{d}(t)^{n d(\rho)} \mid \Delta_{X, \chi}(t)$ and (2) implies that $d(\chi)$ is the multiplicity of $\phi_{2}(t)=(t+1)$ as a factor of $\Delta_{X, \chi}(t)$, where $\phi_{k}(t)$ denotes the cyclotomic polynomial of order $k$. 
See Sections 4.1, 4.2, and 5.1 for the required definitions. The Hodge theoretical condition that 2 be the only weight of $\chi$ can be characterized as the requirement of the equality of the first Betti numbers of both the double cover of $\mathcal{X}$ defined by $\chi$ and its smooth compactification; see Theorem 1.4 for another characterization. In Section 5 we specialize these results to the case of complements of plane curves. The group $\pi_{1}\left(\mathbb{P}^{2} \backslash D\right)$ is closely related to its central extension $\pi_{1}\left(\mathbb{C}^{2} \backslash D\right)$ where $\mathbb{C}^{2}$ is obtained from $\mathbb{P}^{2}$ by deleting a generic line at infinity. In this case the group of characters is isomorphic to $\left(\mathbb{C}^{*}\right)^{r}$, where $r$ is the number of irreducible components of $D$. Moreover, each coordinate codimension-one subtorus can be viewed as the group of characters on $\pi_{1}\left(\mathbb{P}^{2} \backslash D^{i}\right)$, where $D^{i}:=\cup_{j \neq i} D_{j}$; such characters are called coordinate characters. Note that there is a natural inclusion $V_{1}\left(\mathbb{P}^{2} \backslash D^{i}\right) \subset V_{1}\left(\mathbb{P}^{2} \backslash D\right)$ of the coordinate subtori. A character in $V_{1}\left(\mathbb{P}^{2} \backslash D\right)$ is called essential if it is not in any $V_{1}\left(\mathbb{P}^{2} \backslash D^{i}\right)$.

Noncoordinate essential characters are well understood. An algorithm is provided in [26] by the third author to recover such characters as well as their depth from dimensions of linear systems associated with the singularities of $D$. However, much less is known about essential coordinate characters. In Section 5.1 we give the following Hodge theoretical characterization of them.

Theorem 1.4. Let $X=\mathbb{C}^{2} \backslash D$ be the complement of a plane curve $D$. A character in the characteristic variety of $\pi_{1}(X)$ is essential and coordinate if and only if it has weight 2 .

In the case of plane curves the orbifold pencils correspond to solutions of certain equations over the function field $\mathbb{C}(x, y)$. For example, as mentioned in Theorem 1.2, the depths of characters of order 6 are related to the number of independent polynomial solutions in $u, v, w$ of the quasitoric equation $u^{2}+v^{3}=w^{6} F$ of type $(2,3,6)$. This also can be used to relate the cohomology of the Milnor fiber of arrangements of lines with triple points and solutions to the Catalan equation (see [28]).

A similar result for characters of order 2 is shown in Section 5.2. Let $D \subset \mathbb{P}^{2}$ be a projective plane curve, $X_{D}:=\mathbb{P}^{2} \backslash D$ its complement, and $\chi$ a 2-torsion character on $\pi_{1}\left(X_{D}\right)$. Denote by $Q_{(D, \chi)}$ the set of $(2,2,0)$-quasitoric relations associated with $\chi$, that is,

$$
Q_{(D, \chi)}:=\left\{(f, g, h, U, V) \in \mathbb{C}[x, y, z]^{5}\left|f U^{2}-g V^{2}=h, f \cdot g=F, h_{\text {red }}\right| H\right\} / \sim,
$$

where $D:=\{F H=0\}, \chi$ is ramified exactly along $F=0$ (see Definition 5.7) and $\sim$ is the appropriate equivalence relation. Then

Theorem 1.5. The set of $(2,2,0)$-quasitoric relations $Q_{(D, \chi)}$ has a structure of a finitely generated abelian group and

$$
\operatorname{rank} Q_{(D, \chi)} \leq d(\chi)
$$

Moreover, if $X_{D}$ and $\chi$ satisfy the conditions of Theorem $1.3(2)$, then

$$
\operatorname{rank} Q_{(D, \chi)}=d(\chi)
$$


We refer to Section 5.2 for the exact definition of $Q_{(D, \chi)}$ and the equivalence between quasitoric relations. This result is illustrated both in Section 6 of this paper and in [5] with several nontrivial examples aiming to describe a method for calculating the group structure of $Q_{(D, \chi)}$.

Finally, we note that there is a surprising connection between the polynomial equations considered in (1.5) and the Pell equations over the field of rational functions $\mathbb{C}(x, y)$. Investigations of the Pell equations

$$
u^{2}-f(x) v^{2}=1
$$

over the function field $\mathbb{C}(x)$ apparently go back to Abel [1] (see [34]). More recently, the equation (1.6) over $k[x]$ was considered by F. Hazama in [19], [20], where a group structure closely resembling the one described in Theorem 1.5 also appeared. A more detailed study of this connection is out of the scope of this note, but will appear elsewhere.

\section{Characters of fundamental groups}

\subsection{Characteristic varieties}

We recall the basic definitions and results related to characteristic varieties and homology of covering spaces. We will follow the original exposition given in [26], but rephrase it in a more general setting.

Throughout this section $X$ will be a topological space of finite type (that is, $X$ has the homotopy type of a finite $C W$-complex) and $\pi_{1}^{\prime}(X) \subset \pi_{1}(X)$ will be the commutator of its fundamental group. We shall assume that $\pi_{1}(X) / \pi_{1}^{\prime}(X)=$ $H_{1}(X, \mathbb{Z})$ is a free abelian group of rank $r$. Basic examples are complements of plane algebraic curves in $\mathbb{C}^{2}$ with $r$ components and links in a 3-sphere with $r$ components.

Consider the torus of characters of $\pi_{1}(X)$, i.e.,

$$
\operatorname{Char}(X):=\operatorname{Hom}\left(\pi_{1}(X), \mathbb{C}^{*}\right) .
$$

Alternatively, since Char $(X)$ depends only on $\pi_{1}(X)$ we refer to it as $\operatorname{Char}\left(\pi_{1}(X)\right)$. Analogously, we define $\operatorname{Char}(G)$ for a group $G$. The torus $\operatorname{Char}(X)$ is canonically isomorphic to the spectrum $\operatorname{Spec} \mathbb{C}\left[H_{1}(X, \mathbb{Z})\right]=\left(\mathbb{C}^{*}\right)^{r}$ of the group ring of the abelianization of $\pi_{1}(X)$. Let $X_{\mathrm{ab}} \rightarrow X$ be the universal abelian cover, i.e., the covering with the group $H_{1}(X, \mathbb{Z})$. The group $H_{1}(X, \mathbb{Z})$ acts on $X_{\text {ab }}$ as a group of automorphisms and this provides $H_{*}\left(X_{\mathrm{ab}}, \mathbb{C}\right)$ with a structure of a $\mathbb{C}\left[H_{1}(X, \mathbb{Z})\right]$ module. Recall that with each $R$-module $M$ over a commutative ring $R$ one associates the support which is the subvariety of $\operatorname{Spec} R$ consisting of the prime ideals $\mathfrak{p}$ such that the localization $M_{\mathfrak{p}}$ does not vanish.

Definition 2.1. The characteristic variety $V_{k}(X)$ is the subvariety of the torus $\operatorname{Char}(X)=\operatorname{Spec} \mathbb{C}\left[H_{1}(X, \mathbb{Z})\right]$ given as the support of the module $\bigwedge^{k}\left(H_{1}\left(X_{\mathrm{ab}}, \mathbb{C}\right)\right)$ (the exterior power of the homology module). Alternatively, $V_{k}(X)$ can be given 
as the zero set of the $k$-th Fitting ideal of $H_{1}\left(X_{\mathrm{ab}}, \mathbb{C}\right)$, that is, the ideal generated by the $(n-k) \times(n-k)$ minors of the matrix of the map $\Phi$ with coefficients in $\mathbb{C}\left[\pi_{1}(X) / \pi_{1}^{\prime}(X)\right]$

$$
\mathbb{C}\left[\pi_{1}(X) / \pi_{1}^{\prime}(X)\right]^{m} \stackrel{\Phi}{\longrightarrow} \mathbb{C}\left[\pi_{1}(X) / \pi_{1}^{\prime}(X)\right]^{n} \longrightarrow H_{1}\left(X_{\mathrm{ab}}, \mathbb{C}\right) \longrightarrow 0 .
$$

We denote by $\stackrel{\circ}{V}_{k}(X)$ the set of the characters in $V_{k}(X)$ which do not belong to $V_{j}(X)$ for $j>k$. If a character $\chi$ belongs to $\stackrel{\circ}{V}_{k}(X)$, then $k$ is called the depth of $\chi$ and is denoted by $d(\chi)$.

Theorem 2.3 provides a geometric comparison of this definition and comments after (1.1). The following expresses the homology of finite abelian covers in terms of the depths of characters of $\pi_{1}$. The argument follows closely the one given in [25] and [26], but we will present some details here since the statement of Theorem 2.2 is in a more general context than in the references above. See also [6], [21], [31].

Theorem 2.2. Let $X$ be a finite $C W$-complex, let $H$ be a subgroup of $\pi_{1}(X)$ of finite index containing the commutator $\pi_{1}^{\prime}(X)$, and let $K:=\pi_{1}(X) / H$.

Let $i_{H}: \operatorname{Char}(K) \rightarrow \operatorname{Char}(X)$ be the embedding of character varieties induced by the surjection $\pi_{1}(X) \rightarrow K$. Let $X_{H}$ be the covering of $X$ corresponding to the subgroup $H$.

Then

$$
b_{1}\left(X_{H}\right)=b_{1}(X)+\sum_{\xi \in \operatorname{Char}(K) \backslash\{1\}} d\left(i_{H}(\xi)\right) .
$$

Proof. Consider the five term exact sequence corresponding to the spectral sequence

$$
E_{2}^{p, q}=H_{p}\left(L, H_{q}\left(X_{\mathrm{ab}}, \mathbb{C}\right)\right) \Longrightarrow H_{p+q}\left(X_{H}, \mathbb{C}\right),
$$

which is the spectral sequence for the free action of the group $L:=H / \pi_{1}^{\prime}(X)$ on the universal abelian cover $X_{\mathrm{ab}}$. It yields

$$
H_{2}(L, \mathbb{C}) \longrightarrow H_{1}\left(X_{\mathrm{ab}}\right)_{L} \longrightarrow H_{1}\left(X_{H}\right) \longrightarrow H_{1}(L, \mathbb{C}) \longrightarrow 0
$$

(the subscript in second left term denotes covariants). Next, after taking the tensor product of the sequence $(2.2)$ with the group ring $\mathbb{C}\left[H / \pi_{1}^{\prime}(X)\right]=\mathbb{C}[L]$, using for a $\mathbb{C}[L]$-module $M$ the identification of the covariants $M_{L}$ with $M \otimes_{\mathbb{C}[L]} \mathbb{C}$ applied to the second term in 2.5, and finally using the isomorphism

$$
\left(\mathbb{C}\left[\pi_{1}(X) / \pi_{1}^{\prime}(X)\right]\right)^{s} \otimes_{\mathbb{C}[L]} \mathbb{C}=\left(\mathbb{C}\left[\pi_{1}(X) / \pi_{1}^{\prime}(X)\right] / I_{\mathbb{C}[L]}\right)^{s}=(\mathbb{C}[K])^{s}
$$

(here $I_{\mathbb{C}[L]}$ is the augmentation ideal) one obtains

$$
\mathbb{C}[K]^{m} \longrightarrow \mathbb{C}[K]^{n} \longrightarrow H_{1}\left(X_{\mathrm{ab}}, \mathbb{C}\right)_{L} \longrightarrow 0 .
$$


Since Spec $\mathbb{C}[K]$ is canonically identified with $\operatorname{Char}(K)$ and the dimension of the cokernel of the left homomorphism in (2.7) is the sum of the dimensions of the cokernels of localizations of (2.7) at the maximal ideal of $\xi \in \operatorname{Char}(K) \subset \operatorname{Char}(X)$, the dimension of the cokernel in $(2.7)$ is equal to $\sum_{\xi \in \operatorname{Char}(K)} d\left(i_{H}(\xi)\right)$. To conclude the proof we will show that contribution of the character $\xi=1$ in the last sum is equal to the dimension of the image of the left homomorphism in (2.5) and that the right term in (2.5) is equal to $b_{1}(X)$. Indeed, since $b_{1}(X)=\operatorname{rank} \pi_{1}(X) / \pi_{1}^{\prime}(X)$, $\operatorname{dim} H_{1}(L, \mathbb{C})=\operatorname{rank} L$, and the group $K$ is finite, the second claim follows. The first claim follows from consideration of the commutative square obtained by taking the morphism of the sequence (2.5) into the similar five term sequence replacing $H$ by $\pi_{1}(X)$ :

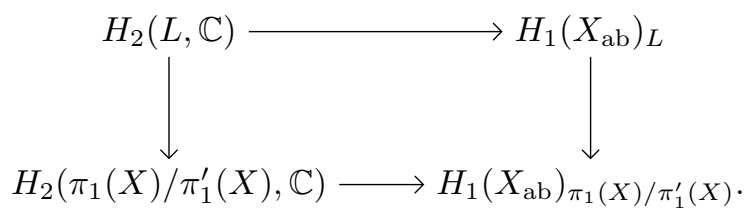

The left vertical arrow is an isomorphism (again since $K$ is finite) and the right vertical arrow is a surjection which is the isomorphism over the contribution of the trivial character in $H_{2}(L, \mathbb{C})$. Hence the identity $(2.3)$ has been verified.

Definition 2.1 allows algorithmic calculation of characteristic varieties (using Fox calculus) provided a presentation of the fundamental group is known. See for example [5] or [36] for explicit examples of such calculations.

On the other hand one has the following interpretation using local systems ([21], [26]). Recall that a (rank $n$ ) local system is a ( $n$-dimensional) linear representation of the fundamental group $\pi_{1}(X)$. For treatment of local systems and their cohomology we refer to [13].

A topological definition of the cohomology of rank one local systems can be given as follows. If $X$ is a finite $C W$-complex, $\chi$ is a character of $\pi_{1}(X)$, and $X_{\mathrm{ab}}$ is the universal abelian cover, then one can define the twisted cohomology $H^{k}(X, \chi)$ as the cohomology of the complex

$$
\cdots \longrightarrow C^{k}\left(X_{\mathrm{ab}}\right) \otimes_{\mathbb{C}\left[H_{1}(X, \mathbb{Z})\right]} \mathbb{C}_{\chi} \stackrel{\partial^{k} \otimes 1}{\longrightarrow} \cdots
$$

where $C^{k}\left(X_{\mathrm{ab}}\right)$ is the $\mathbb{C}$-vector space of $i$-cochains of $X_{\mathrm{ab}}$ considered as a module over the group ring of $H_{1}(X, \mathbb{Z})$ and the $\mathbb{C}_{\chi}$ is the one dimensional $\mathbb{C}$-vector space with the $\mathbb{C}\left[H_{1}(X, \mathbb{Z})\right]$-module structure given by the character $\chi$. If $X$ is a smooth manifold, $H^{*}(X, \chi)$ has a de Rham description (see [13]). The homology of a local system can be described using the dual chain complex. We have the following.

Theorem 2.3. If $\chi \neq 1$, then

$$
d(\chi)=\operatorname{dim} H^{1}(X, \chi)
$$


The connection with the cohomology of local systems allows one to apply general techniques for cohomology with twisted coefficients, which yield the following results on the structure of characteristic varieties.

Theorem $2.4([3])$. Each $V_{k}(X)$ is a finite union of cosets of subgroups of the torus $\operatorname{Char}(X)$. Moreover, for each component $V$ of $V_{k}(X)$ having positive dimension, there is a map $f: X \rightarrow C$, where $C$ is a quasi-projective curve such that $V$ is a coset of the subgroup $f^{*} H^{1}\left(C, \mathbb{C}^{*}\right) \subset \operatorname{Char}(X)$.

Theorem 2.5 ([7]). Let $V$ be an irreducible component of $V_{k}(X)$. Then one of the two following statements holds:

(1) There exists an orbicurve $\mathcal{C}$, a surjective orbifold pencil $f: X \rightarrow \mathcal{C}$, and an irreducible component $W$ of $V_{k}^{\mathrm{orb}}(\mathcal{C})$ such that $V=f^{*}(W)$.

(2) $V$ is an isolated torsion point not of type (1).

Recall the definition of $V_{k}^{\text {orb }}$ after Lemma 1.1 and see Section 4.1 for more details on $V_{k}^{\text {orb }}(\mathcal{C})$ for orbicurves.

\subsection{Alexander polynomial associated with a character}

A specialization of the characteristic variety of a topological space $X$ of finite type to a special character of its fundamental group $G:=\pi_{1}(X)$ can be defined and it is a natural generalization of the Alexander polynomial to this context. For the sake of simplicity, as at the beginning of Section 2.1, we shall assume that an identification $G / G^{\prime} \cong \mathbb{Z}^{r}$ has been made.

Let $X$ be a finite $C W$-complex and let $\chi \in \operatorname{Char}(G)=\left(\mathbb{C}^{*}\right)^{r}$ be a torsion character, where the identification depends on the choice of generators of $H_{1}(X, \mathbb{Z})$. That is $\chi:=\left(\xi_{d}^{\varepsilon_{1}}, \ldots, \xi_{d}^{\varepsilon_{r}}\right)$, where $\xi_{d}$ is a primitive $d$-th root of unity, $0 \leq \varepsilon_{i}<d$, and $d$ is the order of $\chi$. Note that $\chi$ determines naturally an epimorphism $\varepsilon$ : $G / G^{\prime}=\mathbb{Z}^{r} \rightarrow \mathbb{Z}$ defined by $\varepsilon\left(e_{i}\right):=\varepsilon_{i}$. Let $K_{\varepsilon}=\operatorname{ker} \varepsilon$ and let $K_{\varepsilon}^{\prime}=\left[K_{\varepsilon}, K_{\varepsilon}\right]$ be the commutator of $K_{\varepsilon}$. By the Hurewicz theorem, $M_{\chi}:=K_{\varepsilon} / K_{\varepsilon}^{\prime}$ can be identified with the homology of the infinite cyclic cover of $X$ corresponding to $\varepsilon$ and hence it can be viewed as a module over the group $\operatorname{ring} \Lambda:=\mathbb{Q}\left[t^{ \pm 1}\right]$, where $t$ is a generator of the Galois group of covering transformations.

Definition 2.6. Let $X$ and $\chi$ be as above, then the Alexander polynomial of $X$ associated with $\chi$ is a generator of the order of the module $M_{\chi}$ and will be denoted by $\Delta_{X, \chi}(t)$.

The following is a direct consequence of the definition and Theorem 2.3.

Proposition 2.7. Under the above conditions, if $\chi \neq 1$, then $d(\chi)$ is the multiplicity of the factor $\phi_{d}(t)$ in $\Delta_{X, \chi}(t)$, where $\phi_{d}(t)$ is the cyclotomic polynomial of order $d$. 
Proof. Using the same arguments as in Theorem 2.26 of [9], the polynomial $\Delta_{X, \chi}$ is the order of the torsion of

$$
\left(G^{\prime} / G^{\prime \prime} \otimes \mathbb{Q}\right) \otimes_{\Lambda_{r}} \Lambda
$$

where

$$
\Lambda_{r}:=\mathbb{Q}\left[t_{1}^{ \pm 1}, \ldots, t_{r}^{ \pm 1}\right]
$$

and the $\Lambda_{r}$-module structure of $\Lambda$ comes from the identifications $t_{i} \equiv t^{\varepsilon\left(\gamma_{i}\right)}, i=$ $1, \ldots, r$. Therefore $d(\chi)$ is the multiplicity of $\xi_{d}$ as a root of $\Delta_{X, \chi}$. Since $\Delta_{X, \chi} \in$ $\mathbb{Q}[t]$, the result follows.

\subsection{Weight of a character}

Now let us assume that $X$ is a smooth quasi-projective variety and let $\chi \in \operatorname{Char}(X)$ be a character of finite order. Let $X_{\chi}$ be the covering space corresponding to ker $\chi \subset \pi_{1}(X)$. Then $H^{1}\left(X_{\chi}\right)$ supports a mixed Hodge structure with weights 1,2 (see [14], [15]). The cyclic group $\operatorname{Im}(\chi)$ acts (freely) on $X_{\chi}$ preserving both the Hodge and weight filtrations.

Definition 2.8. Let $G$ be a group acting linearly on a complex vector space $V$ and let $\chi \in \operatorname{Char}(G)$. The $\chi$-eigenspace of this action is defined as

$$
V_{\chi}:=\{v \in V \mid g \cdot v=\chi(g) v \forall g \in G\} .
$$

Definition 2.9. An integer $w$ is called a weight of a character $\chi$ if the $\chi$-eigenspace of $\pi_{1}(X)$ acting on $\operatorname{Gr}_{w}^{W} H^{1}\left(X_{\chi}\right)$ has positive dimension. Similarly, $p$ is called a Hodge filtration of $\chi$ if the $\chi$-eigenspace of $\pi_{1}(X)$ acting on $\operatorname{Gr}_{F}^{p} H^{1}\left(X_{\chi}\right)$ has a positive dimension.

The following gives an expression for the weight of a character in terms of finite coverings with arbitrary Galois groups.

Proposition 2.10. Let $X_{G} \rightarrow X$ be an abelian cover of $X$ with a finite covering group $G$. Let $\chi \in \operatorname{Char}(G)$. Then

$$
\operatorname{dim}\left(W_{w} H^{1}\left(X_{G}\right)\right)_{\chi}=\operatorname{dim} W_{w} H^{1}\left(X_{\chi}\right) .
$$

In particular $G$ has a nonzero $\chi$-eigenspace on $W_{w} H^{1}\left(X_{G}\right)$ if and only if $\chi$ has weight $w$.

Proof. The argument is similar to the one in the proof of Theorem 2.2.

Remark 2.11. Note that characters might have either no weights or more than one weight. In this paper we will be most interested in characters with only one weight, namely weight 2 . 


\subsection{Essential, nonessential, and essential-coordinate components}

The constructions described in the previous section can be applied to the case when $X=\mathbb{P}^{2} \backslash \bigcup_{i=0}^{r} D_{i}$, where the $D_{i}$ are irreducible curves. In this case $H_{1}(X, \mathbb{Z})=$ $\mathbb{Z}^{r+1} /\left(d_{0}, \ldots, d_{r}\right)$, where $d_{i}=\operatorname{deg} D_{i}$. Suppose the degree of one of its components, say $D_{0}$, is equal to one, i.e., we have the complement to a plane curve in $\mathbb{C}^{2}$. Then $H_{1}(X, \mathbb{Z})$ is a free abelian group of rank $r$. Let $D=\bigcup D_{i}$ denote the (reducible) curve in $\mathbb{C}^{2}$ formed by irreducible components $D_{i}$. One has a preferred surjection $\pi_{1}\left(\mathbb{C}^{2} \backslash D\right) \rightarrow \mathbb{Z}^{r}$ given by the linking numbers of a loop representing the element of $\pi_{1}$ with the component $D_{i}$ :

$$
\gamma \mapsto\left(\ldots, \operatorname{lk}\left(\gamma, D_{i}\right), \ldots\right)
$$

This also yields the identification $\operatorname{Char}\left(\mathbb{C}^{2} \backslash D\right)=\left(\mathbb{C}^{*}\right)^{r}$.

Let $D^{\prime}$ be a reducible subcurve of $D$ in $\mathbb{C}^{2}$, that is $D^{\prime} \subset D$. Then (see [26]) one has a surjection $\pi_{1}\left(\mathbb{C}^{2} \backslash D\right) \rightarrow \pi_{1}\left(\mathbb{C}^{2} \backslash D^{\prime}\right) \rightarrow 1$ and hence an embedding

$$
i_{D^{\prime}}: \operatorname{Char}\left(\mathbb{C}^{2} \backslash D^{\prime}\right) \rightarrow \operatorname{Char}\left(\mathbb{C}^{2} \backslash D\right) .
$$

The image of $i_{D^{\prime}}$ is formed by the factors of $\left(\mathbb{C}^{*}\right)^{r}$ corresponding to the components of $D^{\prime}$. Moreover it was shown in [26] that if $\chi \in V_{k}\left(\mathbb{C}^{2} \backslash D^{\prime}\right)$ then $i_{D^{\prime}}(\chi) \in V_{j}$ with $j \geq k$.

Definition 2.12 ([26]). The components of the characteristic variety $V_{k}(D)$ obtained as the image of a component of $V_{k}\left(D^{\prime}\right)$ are called nonessential. A component of $V_{k}$ is called coordinate if it belongs to $i_{D^{\prime}}\left(\operatorname{Char}\left(\mathbb{C}^{2} \backslash D^{\prime}\right)\right)$ for some $D^{\prime} \subsetneq D$.

Recall that a meridian of a component $D_{i} \subset D$ is the class of the positively oriented boundary $\gamma_{i} \in \pi_{1}\left(\mathbb{C}^{2} \backslash D\right)$ of a small disk transversal to $D_{i}$ up to conjugacy (this terminology is also applied to its homology class).

Definition 2.13. A character $\chi \in \operatorname{Char}\left(\mathbb{C}^{2} \backslash D\right)$ is unramified along a component $D_{i} \subset D$ if for a meridian $\gamma_{i} \in H_{1}\left(\mathbb{C}^{2} \backslash D\right)$ of $D_{i}$ one has $\chi\left(\gamma_{i}\right)=1$.

This terminology is consistent with the notion of ramification of a character on a prime ideal over the ring of integers of a number field.

Remark 2.14. If $G$ is a quotient of $\pi_{1}\left(\mathbb{C}^{2} \backslash D\right)$ then $\operatorname{Char}(G)$ is naturally a subset of $\operatorname{Char}\left(\mathbb{C}^{2} \backslash D\right)$ and this definition also applies. Note also that the characters of $\pi_{1}\left(\mathbb{C}^{2} \backslash D\right)$ unramified along $D_{i}$ can be identified with the characters of $\pi_{1}\left(\mathbb{C}^{2} \backslash D^{i}\right)$ where $D^{i}$ is the union of all irreducible components of $D$ other than $D_{i}$.

Given a surjection $\pi_{1}\left(\mathbb{C}^{2} \backslash \bigcup C_{k}\right) \rightarrow G:=\mathbb{Z}_{a_{1}} \oplus \cdots \oplus \mathbb{Z}_{a_{m}}$ (for $m \leq r$ ), one can construct the unbranched covering space $X_{a_{1}, \ldots, a_{m}}$ corresponding to the kernel of the above surjection of the fundamental group. Moreover, there is a compactification of this unbranched cover and its morphism to $\mathbb{P}^{2}$ extending the covering map. Though this compactification (branched cover) is nonunique, its birational class is well defined. In particular the first Betti number of this branched cover 
is well defined. A compactification $\bar{X}_{a_{1}, \ldots, a_{m}}$ can be selected so that it supports a $G$-action extending the action of $G$ on $X_{a_{1}, \ldots, a_{m}}$. A calculation of $H_{1}\left(\bar{X}_{a_{1}, \ldots, a_{m}}, \mathbb{C}\right)$ as a $G$-module is given by the following.

Theorem 2.15 ([31]). For each character $\chi$ of $G=\mathbb{Z}_{a_{1}} \oplus \cdots \oplus \mathbb{Z}_{a_{m}}$, let $V_{\chi}$ be the $\chi$-eigenspace of the $G$-action on $H_{1}\left(\bar{X}_{a_{1}, \ldots, a_{m}}, \mathbb{C}\right)$. Let $D_{\chi}$ be the union of the components of $D$ over which the character $\chi$ ramifies. Then $\operatorname{dim} V_{\chi}$ is equal to the depth of $\chi$ considered as the character of $\pi_{1}\left(\mathbb{C}^{2} \backslash D_{\chi}\right)$.

This theorem was used in [26] to describe essential components of $V_{k}(D)$ in terms of combinatorics of singularities of $D$ and the superabundances of the linear systems of curves given by the local type of singularities, their position on $\mathbb{P}^{2}$ and the degree of $D$.

\section{Albanese varieties of smooth quasi-projective varieties.}

Recall (see [33]) that given a projective variety $X$ there is a canonically associated abelian variety $\operatorname{Alb}(X)$ and the map $X \rightarrow \operatorname{Alb}(X)$ (unique up to a choice of the image of point in $X$ ) is universal with respect to maps into abelian varieties, i.e., given an abelian variety $A$ and a morphism $X \rightarrow A$ there is a unique (up to ambiguity, as above) factorization $X \rightarrow \operatorname{Alb}(X) \rightarrow A$.

This construction can be extended to the quasi-projective case so that the Albanese variety is a semiabelian variety which is universal with respect to morphisms into algebraic groups. For example one can use Deligne's construction of the 1-motif associated with the mixed Hodge structure on cohomology $H^{1}(X)$ of a smooth quasi-projective variety (see [13]). More precisely one has the following.

Theorem 3.1. Let $X$ be a quasi-projective variety which is a complement to a divisor with normal crossings in a smooth projective variety $\bar{X}$.

(1) Then one has an exact sequence

$$
0 \rightarrow \mathcal{A} \rightarrow \operatorname{Alb}(X) \rightarrow \operatorname{Alb}(\bar{X}) \rightarrow 0
$$

where $\mathcal{A}$ is an affine abelian algebraic group isomorphic to a product of copies of the multiplicative group $\mathbb{G}_{m} \cong \mathbb{C}^{*}$. The semiabelian variety $\operatorname{Alb}(X)$ depends on $X$ functorially, i.e., a morphism $X_{1} \rightarrow X_{2}$ induces a homomorphism $\operatorname{Alb}\left(X_{1}\right) \rightarrow \operatorname{Alb}\left(X_{2}\right)$.

(2) If $\Gamma$ is a finite group of biholomorphic automorphisms of $X$ then the sequence (3.1) is compatible with the action of $\Gamma$.

An explicit construction can be given as follows (see also [22]). Let $\bar{X}$, as above, be a smooth compactification of $X$ such that $\bar{X} \backslash X=D=\bigcup D_{i}$ is a union of smooth divisors having normal crossings. Then

$$
\operatorname{Alb}(X)=H^{0}\left(\bar{X}, \Omega_{\bar{X}}^{1}(\log D)\right)^{*} / H_{1}(X, \mathbb{Z}),
$$


where the embedding of $H_{1}(X, \mathbb{Z})$ as a lattice is given by $\gamma(\omega):=\int_{\gamma}(\omega)$. The Albanese map is given by

$$
P \mapsto \int_{P_{0}}^{P} \omega
$$

(here $P_{0}$ is a fixed point on $X$ ). The integral (3.3) depends (modulo periods of $\omega$ ) only on the end points of the path since a holomorphic logarithmic form is closed (see [13]). One has the commutative diagram

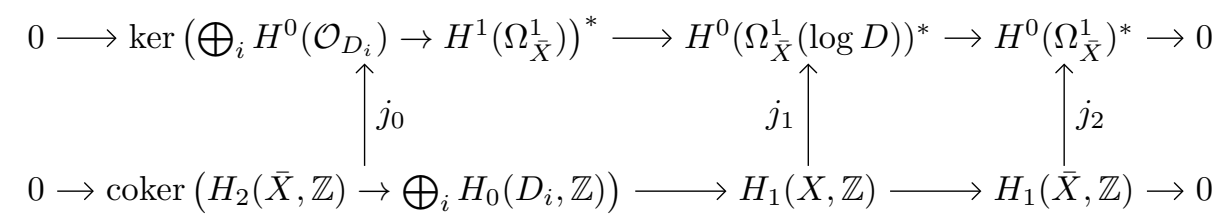

In this diagram the upper row is dual to the exact cohomology sequence corresponding to the sequence of sheaves given by the residue map (see (3.1.5.2) in [13]),

$$
0 \longrightarrow \Omega_{\bar{X}}^{1} \longrightarrow \Omega_{\bar{X}}^{1}(\log D) \stackrel{\oplus_{i} \operatorname{Res}_{D_{i}}}{\longrightarrow} \bigoplus_{i} j_{*} \mathcal{O}_{D_{i}} \longrightarrow 0
$$

where $j: D_{i} \rightarrow \bar{X}$. The lower row is the exact sequence of the pair $(\bar{X}, X)$ in which we use the identification

$$
H_{2}(\bar{X}, X, \mathbb{Z})=H^{2 \operatorname{dim} D}(D, \mathbb{Z})=\bigoplus_{i} H^{2 \operatorname{dim} D}\left(D_{i}, \mathbb{Z}\right)=\bigoplus_{i} H_{0}\left(D_{i}, \mathbb{Z}\right)
$$

One can check that all vertical arrows are injective and the image of each provides the lattice in the corresponding complex vector space in the upper row. The rank of the lattice which is the image of $j_{2}$ (resp. $j_{0}$ ) is equal to the real (resp. complex) dimension of the target. Note that the fact that one uses the real dimension of $H^{0}\left(\Omega_{\bar{X}}^{1}\right)$ is that $\operatorname{rank} H_{1}(X, \mathbb{Z})=\operatorname{dim}_{\mathbb{C}} H^{1}\left(\mathcal{O}_{\bar{X}}\right)+\operatorname{dim} H^{0}\left(\Omega_{\bar{X}}^{1}(\log D)\right)$ which follows from the degeneration of the Hodge-de Rham spectral sequence in the $E_{1}$-term.

In the case of $j_{0}$, one uses that the map dual to $H_{2}(\bar{X}, \mathbb{C}) \rightarrow \bigoplus_{i} H_{0}\left(D_{i}, \mathbb{C}\right)$ factors as

$$
\bigoplus_{i} H^{0}\left(D_{i}, \mathbb{C}\right) \rightarrow H^{1,1}(\bar{X}) \rightarrow H^{2}(\bar{X}, \mathbb{C})
$$

Hence the quotient of the right (resp. left) injection is a complex torus (resp. affine algebraic group isomorphic to a product of several copies of $\mathbb{C}^{*}$ ). This complex torus is isomorphic to the Albanese variety of $\bar{X}$ by the classical construction. The remaining assertions of the Theorem 3.1 follow from the description of the Albanese map given by (3.3).

Finally note that Theorem 3.1 implies the following. 
Corollary 3.2. Let $\phi$ be an involution of $X$ and let $\phi_{*}$ be the corresponding automorphism of $\operatorname{Alb}(X)$. Let $\operatorname{Alb}(X)^{-}=\left\{v \in \operatorname{Alb}(X) \mid i \phi_{*}(v)=-v\right\}$ and $\operatorname{Alb}(X)^{+}=\left\{v \in \operatorname{Alb}(X) \mid i \phi_{*}(v)=v\right\}$. Then one has an isogeny

$$
\operatorname{Alb}(X)=\operatorname{Alb}(X)^{-} \oplus \operatorname{Alb}(X)^{+} .
$$

\section{Orbifold pencils and characters of fundamental groups of quasi-projective manifolds}

\subsection{Orbicurves}

\section{Definitions 4.1.}

1. An orbicurve $\mathcal{C}$ is a complex one-dimensional orbifold, i.e., a smooth complex curve with a finite collection $\mathcal{R}$ of points (called the orbifold points) with a multiplicity assigned to each point in $\mathcal{R}$. The complement to $\mathcal{R}$ is called the regular part of the orbifold and is denoted by $\mathcal{C}_{\text {reg }}$.

2. An orbicurve $\mathcal{C}$ is called a global quotient if there exist a finite group $G$ and a manifold $C$ with a $G$-action such that $\mathcal{C}$ is the quotient of $C$ by $G$ with the standard orbifold structure. The group $G$ will be called the covering group of the global quotient. If $R$ is the preimage of $\mathcal{R}$ via the covering, then $C \backslash R \rightarrow \mathcal{C} \backslash \mathcal{R}$ is an unramified Galois covering whose deck transformation group is also $G$.

3. (See [2]) The orbifold fundamental group $\pi_{1}^{\text {orb }}(\mathcal{C})$ of an orbifold $\mathcal{C}$ is defined as the quotient $\pi_{1}(\mathcal{C} \backslash \mathcal{R})$ by the normal closure of the elements $\gamma_{p_{i}}^{m\left(p_{i}\right)}$ where $m\left(p_{i}\right)$ is the multiplicity of an orbifold point $p_{i}$ and $\gamma_{i}$ is a meridian of $p_{i}$.

4. A marking of an orbicurve $\mathcal{C}$ (resp. a quasi-projective variety $\mathcal{X}$ ) is a character of its orbifold fundamental group (resp. its fundamental group), that is, an element of $\operatorname{Char}^{\text {orb }}(\mathcal{C}):=\operatorname{Hom}\left(\pi_{1}^{\text {orb }}(\mathcal{C}), \mathbb{C}^{*}\right)(\operatorname{resp} . \operatorname{Char}(X))$. Note that this terminology is different from that used in [2].

5. A marked orbicurve is a pair $(\mathcal{C}, \rho)$, where $\mathcal{C}$ is an orbicurve and $\rho$ is a marking of $\mathcal{C}$. Similarly, one defines a marked quasi-projective manifold as a pair $(X, \chi)$ consisting of a quasi-projective manifold $X$ and a character of its fundamental group; note that for a marked orbicurve $(\mathcal{C}, \rho)$ the pair $\left(\mathcal{C}_{\text {reg }}, \rho_{\text {reg }}\right)$ is a marked quasi-projective manifold where $\rho_{\text {reg }}$ is the character induced by $\rho$ via $\mathcal{C}_{\text {reg }} \hookrightarrow \mathcal{C}$. Henceforth, all characters used as markings will be assumed to have finite order.

6. A marked global quotient is a marked orbicurve $(\mathcal{C}, \rho)$ such that if $C \backslash R \rightarrow$ $\mathcal{C} \backslash \mathcal{R}$ is the unbranched cover corresponding to the global quotient $C \rightarrow \mathcal{C}$, with $R$ being the set of fixed points of nonidentity elements of the covering group $G$, and $\mathcal{R}$ being its image (or equivalently the set of orbifold points), then one has

$$
\pi_{1}(C \backslash R)=\operatorname{ker}\left(\pi_{1}(\mathcal{C} \backslash \mathcal{R}) \rightarrow \pi_{1}^{\text {orb }}(\mathcal{C}) \stackrel{\rho}{\rightarrow} \mathbb{C}^{*}\right) .
$$


In other words, the cover $C \backslash R \rightarrow \mathcal{C} \backslash \mathcal{R}$ is the cover of minimal degree over which $\rho$ becomes trivial.

Remark 4.2. The above existence condition for a marking of a global quotient implies that the quotient map over the regular part of the orbifold is a cyclic cover. More precisely, the covering group $G$ can be identified with $\operatorname{Im}(\rho) \subset \mathbb{C}^{*}$, i.e., $\rho$ can be viewed as a character of the covering group $G$. If $d$ is the order of this covering group then the number of possible markings is equal to the value of the Euler function $\phi(d)$.

Definition 4.3. Let $\mathcal{C}$ be a global orbifold quotient and let $\rho$ be a marking. Let $R$ be the set of orbifold points and let $C \backslash R \rightarrow \mathcal{C} \backslash \mathcal{R}$ be the quotient map with the covering group $G$. The integer

$$
d(\rho)=\operatorname{dim}\left\{v \in H^{1}(C \backslash R, \mathbb{C}) \mid g \cdot v=\rho(g) v, \quad g \in G\right\}
$$

is called the depth of the character $\rho$ of the orbicurve $\mathcal{C}$.

Remark 4.4. In the previous definition, note that the points in $R$ have nontrivial isotropy and thus it is not difficult to check that

$$
d(\rho)=\operatorname{dim}\left\{v \in H^{1}(C, \mathbb{C}) \mid g \cdot v=\rho(g) v, \quad g \in G\right\} .
$$

This definition of the depth of a character for orbicurves will be used in the proof of Theorem 1.3 (see Section 4.3). Moreover, an alternative definition of the depth of $\rho$ is that it be the depth of the character of the finitely presented group $\pi_{1}^{\text {orb }}(\mathcal{C})$. Using the same ideas as in Theorem 2.15, it is easily seen that, for nontrivial characters, these two definitions coincide.

Example 4.5. Let $\mathbb{C}_{n, n}$ be the orbifold supported on $\mathbb{C}$ with two orbifold points of multiplicity $n$. We shall identify $\mathbb{C}$ with $\mathbb{P}^{1} \backslash\{[1: 1]\}$ so that the orbifold points correspond to $[0: 1]$ and $[1: 0]$. This orbifold is the global quotient of a smooth curve $C$ by the cyclic group $\mathbb{Z} / n$ where $C$ is the complement in $\mathbb{P}^{1}$ of the set $S:=\left\{\left[\xi_{n}^{i}: 1\right] \mid i=0,1, \ldots, n-1\right\}$ of $n$ points (here $\xi_{n}$ is a primitive root of unity of degree $n$ ) and the global quotient map is the restriction to the complement of $S$ of the map $\mathbb{P}^{1} \rightarrow \mathbb{P}^{1}$ given by $z \mapsto z^{n}$. We have $\pi_{1}^{\text {orb }}\left(\mathbb{C}_{n, n}\right)=\mathbb{Z} / n * \mathbb{Z} / n$, and hence the group of characters $\operatorname{Char}\left(\pi_{1}^{\text {orb }}\left(\mathbb{C}_{n, n}\right)\right)$ is isomorphic to $\mu_{n} \times \mu_{n}$, where $\mu_{n}$ is the group of $n$-th roots of unity. Consider the character $\rho \in \operatorname{Char}\left(\pi_{1}^{\text {orb }}\left(\mathbb{C}_{n, n}\right)\right)$ taking the values $\zeta$ and $\zeta^{-1}$ on generators of the direct sum, where $\zeta$ is a primitive root of unity. It follows that if $\pi_{1}\left(\mathbb{P}^{1} \backslash\{[1: 0],[1: 1],[0: 1]\}\right) \rightarrow \pi_{1}^{\text {orb }}\left(\mathbb{C}_{n, n}\right)$ is the canonical surjection (using the above identification of $\mathbb{C}$ and $\mathbb{P}^{1}$ so that the point at infinity corresponds to $\left.[1: 1]\right)$, then the pullback of $\rho$ takes the values $\zeta, 1$, and $\zeta^{-1}$ on generators corresponding to $[1: 0],[1: 1]$, and $[0: 1]$. The action on the set $S$ of the covering group $\operatorname{Im}(\rho)$ of the cover $\mathbb{P}^{1} \backslash\{[1: 0],[0: 1]\} \rightarrow \mathbb{P}^{1} \backslash\{[1: 0],[0: 1]\}$ given by $z \rightarrow z^{n}$ is just cyclic permutation of points of $S$. Since $H_{1}\left(\mathbb{P}^{1} \backslash\{[1: 0], S,[0: 1]\}, \mathbb{Z}\right)$ generated by meridians around each of the deleted points with sum of meridians equal to zero being 
the single relation is follows that $H_{1}\left(\mathbb{P}^{1} \backslash\{[1: 0],[1: 1],[0: 1]\}, \mathbb{Z}\right)=\mathbb{Z} \oplus \mathbb{Z}^{n}$ (with each component of the second summand being the meridian about a point of $S$ ). The action of $\mu_{n}=\operatorname{Im}(\rho)$ is a cyclic permutation. Hence $d(\rho)=1$. Moreover, the branched covering space of $\mathbb{C}$ corresponding to such a $\rho$ is $\mathbb{P}^{1} \backslash S$ and the dimension of the $\rho$-eigenspace in $H_{1}$ of the branched cover is one as well (this is a special case of [31]). For an alternative way to obtain this result see Proposition 2.7 in [7].

\subsection{Orbifold pencils}

Definition 4.6. Let $\mathcal{X}$ be a quasi-projective manifold and $\mathcal{C}$ be an orbicurve. A dominant holomorphic map $\phi$ between $\mathcal{X}$ and the underlying complex curve $\mathcal{C}$ is called an orbifold pencil if the index of each orbifold point $p$ divides the multiplicity of each connected component of the fiber $\phi^{*}(p)$ over $p$.

Remark 4.7. Note that this definition implies that if $\Gamma_{i}$ is a meridian of an irreducible component of $\phi^{-1}\left(p_{i}\right)$ then $\phi\left(\Gamma_{i}\right)$ belongs to the subgroup of $\pi_{1}\left(\mathcal{C} \backslash p_{i}\right)$ normally generated by $\gamma_{i}^{m\left(p_{i}\right)}$. In particular an orbifold pencil induces a map $\phi_{*}: \pi_{1}(\mathcal{X}) \rightarrow \pi_{1}^{\text {orb }}(\mathcal{C})$ and the following definition makes sense.

Definition 4.8. Let $(\mathcal{X}, \chi)$ be a marked quasi-projective manifold and let $(\mathcal{C}, \rho)$ be a marked orbicurve. A marked orbifold pencil is an orbifold pencil $\phi: X \rightarrow \mathcal{C}$ such that $\chi=\rho \circ \phi_{*}$.

Definition 4.9. Let $\mathcal{X}$ be a quasi-projective variety, let $C$ be a quasi-projective curve, and let $\mathcal{C}$ be an orbicurve which is a global quotient of $C$. A global quotient orbifold pencil is an orbifold pencil $\phi: \mathcal{X} \rightarrow \mathcal{C}$ such that there exists a morphism $\Phi: X_{G} \rightarrow C$, where $X_{G}$ is a quasi-projective manifold endowed with an action of the group $G$ which makes the diagram

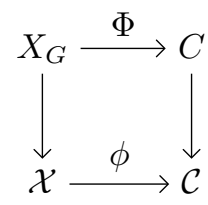

commutative, in which the vertical arrows are the quotients by the action of $G$.

If, in addition, $(\mathcal{X}, \chi)$ and $(\mathcal{C}, \rho)$ are marked, then the global quotient orbifold pencil $\phi: \mathcal{X} \rightarrow \mathcal{C}$ is marked if $\chi=\phi^{*}(\rho)$ where $\phi^{*}: \operatorname{Char}^{\text {orb }}(\mathcal{C}) \rightarrow \operatorname{Char}(\mathcal{X})$ is the homomorphism dual to the surjection $\phi_{*}: \pi_{1}(\mathcal{X}) \rightarrow \pi_{1}^{\text {orb }}(\mathcal{C})$ corresponding to the orbifold pencil $\phi$. We will refer to the map of pairs $\phi:(\mathcal{X}, \chi) \rightarrow(\mathcal{C}, \rho)$ as a marked global quotient orbifold pencil in $(\mathcal{X}, \chi)$ with target $(\mathcal{C}, \rho)$.

Remark 4.10. Consider the collection $\mathcal{R}$ of nonmanifold points in $\mathcal{C}$ and $F$ the collection $\phi^{-1}(\mathcal{R})$ of multiple fibers corresponding to $\phi$. The orbifold relation $\chi=\phi^{*}(\rho)$ holds if and only if there holds

$$
\hat{\chi}=i^{*}(\chi), \quad \hat{\chi}=\hat{\phi}^{*}(\hat{\rho}), \quad \hat{\rho}=p^{*}(\rho),
$$


for the map $\hat{\phi}: \mathcal{X} \backslash F \rightarrow \mathcal{C} \backslash \mathcal{R}$ of open manifolds induced by $\phi$, where $i: \mathcal{X} \backslash F \rightarrow \mathcal{X}$ is the embedding and $p: \pi_{1}(\mathcal{C} \backslash \mathcal{R}) \rightarrow \pi_{1}^{\text {orb }}(\mathcal{C})$ is the canonical projection.

Lemma 4.11. Let $\phi$ be a marked orbifold pencil of $(\mathcal{X}, \chi)$ with target $(\mathcal{C}, \rho)$. If $\mathcal{C}$ is a global quotient of a curve $C$ then $\phi$ is a marked global quotient orbifold pencil, i.e., $\phi$ can be extended to a commutative diagram (4.4).

Proof. As usual, denote by $\mathcal{R} \subset \mathcal{C}$ the set of nonmanifold points of $\mathcal{C}$ and by $F$ its preimage under the morphism $\phi$. Following the notation from Remark 4.10, and the hypothesis that $\phi$ be a marked orbifold pencil, one has $\hat{\chi}=\hat{\phi}^{*}(\hat{\rho})$; the existence of a covering $\pi_{G}: X_{G} \rightarrow \mathcal{X}$ associated with the (finite index) kernel of the homomorphism $\pi_{1}(\mathcal{X} \backslash F) \rightarrow \pi_{1}(\mathcal{X}) \rightarrow \mu_{n}$, where the right homomorphism is the character $\chi$; and finally the commutative diagram

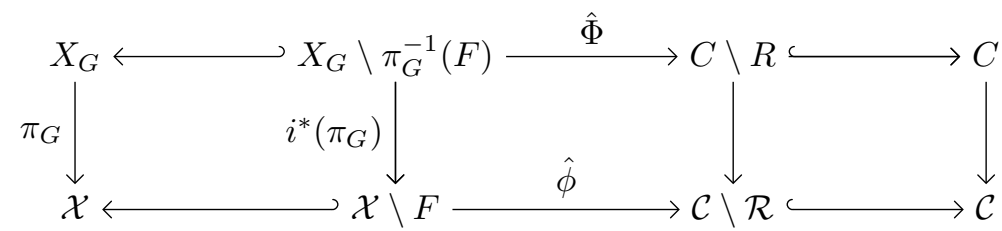

where $i^{*}\left(\pi_{G}\right)$ is the restriction of $\pi_{G}$ to $X_{G} \backslash \pi_{G}^{-1}(F)$.

Since the covering is finite, it is classical that $X_{G}$ can be chosen to be quasiprojective and it is only necessary to construct the extension up to an algebraic subvariety of codimension 2 . We have to verify that $\hat{\Phi}$ extends to a map $\Phi: X_{G} \rightarrow C$. For any $P \in \operatorname{Reg}\left(\pi_{G}^{-1}(F)\right)$, smooth point of $\pi_{G}^{-1}(F)$, the definition of $\Phi(P)$ can be given as follows. Assume $D_{P}$ is a small disc centered at $P$ and transversal to $\pi_{G}^{-1}(F)$. Its (positively oriented) boundary $\gamma_{P}$ defines a meridian of $X_{G} \backslash \pi_{G}^{-1}(F)$. By construction, $i^{*}\left(\pi_{G}\right)\left(\gamma_{P}\right)$ is a meridian in $\pi_{G}(P)$ and $\hat{\phi}\left(i^{*}\left(\pi_{G}\right)\left(\gamma_{P}\right)\right)$ is a meridian in $\phi\left(\pi_{G}(P)\right)$, and thus its preimage by the global quotient map of $\mathcal{C}$ is a meridian around a point of $Q \in R$. We define $\Phi(P)=Q$. This map is easily checked to be well defined by the finiteness of $G$ and Lemma 1.1.

Definition 4.12. Global quotient orbifold pencils $\phi_{i}:(\mathcal{X}, \chi) \rightarrow(\mathcal{C}, \rho), i=1, \ldots, n$ are called independent if the induced maps $\Phi_{i}: X_{G} \rightarrow C$ constructed in Lemma 4.11 define $\mathbb{Z}[G]$-independent morphisms of modules

$$
\Phi_{i *}: H_{1}\left(X_{G}, \mathbb{Z}\right) \rightarrow H_{1}(C, \mathbb{Z}) .
$$

If, in addition, $\bigoplus_{i} \Phi_{i *}: H_{1}\left(X_{G}, \mathbb{Z}\right) \rightarrow H_{1}(C, \mathbb{Z})^{n}$ is surjective, we say that the pencils $\phi_{i}$ are strongly independent.

Remark 4.13. Note that if either $n=1$ or $H_{1}(C, \mathbb{Z})=\mathbb{Z}[G]$, then independence is equivalent to the surjectivity of $\bigoplus_{i} \Phi_{i *} \otimes \mathbb{Q}$. For example, in the latter case,

$$
\bigoplus_{i=1}^{n} \Phi_{i *} \otimes \mathbb{Q} \in \operatorname{Hom}_{\mathbb{Q}[G]}\left(H_{1}\left(X_{G}, \mathbb{Q}\right), \mathbb{Q}[G]^{n}\right)
$$

can be described by a matrix whose columns are the vectors corresponding to $\Phi_{i *}$, $i=1, \ldots, n$. 
This definition is motivated by the following.

Proposition 4.14. Let $\mathcal{C}$ be an orbicurve which is a global $G$-quotient of the algebraic group $\mathcal{A}=\mathbb{C}^{*}$. The global quotient orbifold pencils $\phi_{i}: \mathcal{X} \rightarrow \mathcal{C}, i=1, \ldots, r$, on a global quotient orbifold $\mathcal{X}=X_{G} / G$ such that the first Betti number of a smooth compactification of $X_{G}$ is zero, are independent in the sense of Definition 4.12 if and only if they define $\mathbb{Z}$-independent elements of the abelian group $\operatorname{Mor}_{G}\left(X_{G}, \mathcal{A}\right)$ of equivariant morphisms.

Proof. Note that $\operatorname{Mor}_{G}\left(X_{G}, \mathbb{C}^{*}\right)=\operatorname{Hom}_{G}\left(\operatorname{Alb}\left(X_{G}\right), \mathbb{C}^{*}\right)$ by the universal property of maps from the Albanese variety into an algebraic group. The assumption on the first Betti number of compactification of $X_{G}$ yields that $\operatorname{Alb}\left(X_{G}\right)$ is a torus of dimension $b_{1}\left(X_{G}\right)$. Also $\operatorname{Hom}_{G}\left(\operatorname{Alb}\left(X_{G}\right), \mathbb{C}^{*}\right)=\operatorname{Hom}\left(H_{1}\left(X_{G} ; \mathbb{Z}\right), H_{1}\left(\mathbb{C}^{*} ; \mathbb{Z}\right)\right)$ holds equivariantly and the claim follows.

Remark 4.15. In [11] it was shown that Proposition 4.14 is also true for special $\mathcal{X}$ in cases when $\mathcal{A}$ is a certain elliptic curve (depending on the Alexander polynomial of $\mathcal{X}$ ). This is so if $\mathcal{X}$ is the complement of a cuspidal curve $C$ in $\mathbb{P}^{2}$. In this case $\operatorname{Mor}_{G}\left(X_{G}, \mathcal{A}\right)$ can be identified with the Mordell-Weil group of $K$-points of the elliptic curve over $\mathbb{C}$ admitting an automorphism of order 6 where $K$ is the field of rational functions on the 6 -fold cover of $\mathbb{P}^{2}$ ramified along the curve, i.e., the degree six extension of $\mathbb{C}(x, y)$. This is also the case for the $\delta$-curves discussed in [11].

On the other hand, in the case of orbifolds with trivial orbifold structure there are very few marked orbifold pencils.

Proposition 4.16. Let $\phi_{i}:(\mathcal{X}, \chi) \rightarrow(C, \rho), i=1, \ldots, n$, be a collection of strongly independent marked pencils. Assume that $C$ has a trivial orbifold structure. Then $\rho=1$ and hence $\chi=1$.

Proof. Consider the map $\left(\phi_{1}, \ldots, \phi_{n}\right): \mathcal{X} \rightarrow C^{n}$. Independence implies that the induced map $H_{1}(\mathcal{X}) \rightarrow H_{1}\left(C^{n}\right)$ is surjective and hence the dual map on cohomology is injective. If $p_{i}: C^{n} \rightarrow C$ is the projection on the $i$-th factor, then $p_{i}^{*}(\rho)=(1, \ldots, 1, \rho, 1, \ldots, 1)$ (the nonidentity component is on the $i$-th coordinate). The compatibility condition together with the injectivity of $H^{1}\left(C, \mathbb{C}^{*}\right) \rightarrow H^{1}(\mathcal{X})$ implies that $p_{i}^{*}(\rho)=p_{j}^{*}(\rho)$ and hence $\rho=1$.

\subsection{Orbifold pencils, depth, and roots of Alexander polynomials}

In this section we prove Theorem 1.3.

Proof of Theorem 1.3. In order to prove part (1), we consider $n$ strongly independent orbifold pencils $\phi_{1, *}, \ldots, \phi_{n, *}$. Since

$$
H_{1}\left(X_{G} ; \mathbb{Z}\right) \stackrel{\oplus_{i} \Phi_{i, *}}{\longrightarrow} H_{1}(C ; \mathbb{Z})^{n}
$$


is an equivariant epimorphism, the dual morphism $H^{1}(C ; \mathbb{Z})_{\rho}^{n} \rightarrow H_{1}\left(X_{G} ; \mathbb{Z}\right)_{\chi}$ is injective and hence $\operatorname{rank} H^{1}(C ; \mathbb{Z})_{\rho}^{n}=n d(\rho) \leq \operatorname{rank} H_{1}\left(X_{G} ; \mathbb{Z}\right)_{\chi}=d(\chi)$ (see Theorem 2.15 and Remark 4.4).

As for part (2), let $\chi$ be a 2 -torsion character. We apply the formula for the first Betti number in Theorem 2.2 to the degree 2 covering

$$
X_{\chi} \rightarrow \mathcal{X}
$$

corresponding to the subgroup $\operatorname{ker} \chi$ of $\pi_{1}(\mathcal{X})$; note that $G:=\pi_{1}(\mathcal{X}) / \operatorname{ker} \chi$ is the group of two elements and $X_{\xi}$ has also been denoted by $X_{G}$.

The sum in (2.3) contains only one term and yields, together with Theorem 2.15, that $d(\chi)$ is the dimension of the $\chi$-eigenspace of $H_{1}\left(X_{\chi}, \mathbb{C}\right)$ :

$$
d(\chi)=\left\{v \in H_{1}\left(X_{\chi}, \mathbb{C}\right) \mid g \cdot v=\chi(g) v\right\} .
$$

Moreover, the action of $g \in G, g \neq 1$, is multiplication by -1 . The action of $g$ on $\operatorname{Alb}\left(X_{\chi}\right)$ induces the isogeny $\operatorname{Alb}\left(X_{\chi}\right)=\operatorname{Alb}(X) \oplus \operatorname{Alb}\left(X_{\chi}\right)^{-}$where the second summand is the subvariety of $\operatorname{Alb}\left(X_{\chi}\right)$ comprising points on which the covering group acts as multiplication by -1 (see Corollary 3.2 ; note that $\left.\operatorname{Alb}\left(X_{\chi}\right)^{+}=\operatorname{Alb}(X)\right)$. Since we assume that $\chi$ has only weight $2, \operatorname{Alb}\left(X_{\chi}\right)^{-}$has no compact part. In particular, by Theorem 3.1,

$$
\operatorname{Alb}\left(X_{\chi}\right)^{-}=\operatorname{ker}\left(\operatorname{Alb}\left(X_{\chi}\right) \rightarrow \operatorname{Alb}(X)\right)=\left(\mathbb{C}^{*}\right)^{d(\chi)},
$$

and the order 2 action is given by $\pi_{\rho}: z \mapsto z^{-1}$. Therefore the projections give $d(\chi)$ independent equivariant (due to Albanese functoriality) maps $X_{\chi} \rightarrow \mathbb{C}^{*}$. Since $\operatorname{Mor}_{\mathbb{Z}_{2}}\left(X_{\chi}, \mathbb{C}^{*}\right)=\operatorname{Hom}_{\mathbb{Z}_{2}}\left(\operatorname{Alb}\left(X_{\chi}\right), \mathbb{C}^{*}\right)$ and $\operatorname{Hom}_{\mathbb{Z}_{2}}\left(\left(\mathbb{C}^{*}\right)^{d(\chi)}, \mathbb{C}^{*}\right)=\operatorname{Hom}\left(\mathbb{Z}^{d(\chi)}, \mathbb{Z}\right)$, then $\operatorname{rank}_{\mathbb{Z}} \operatorname{Mor}_{\mathbb{Z}_{2}}\left(X_{\chi}, \mathbb{C}^{*}\right)=d(\chi)$. Hence each map descends to an orbifold pencil $\mathcal{X} \rightarrow \mathbb{C}_{2,2}$. Then one has the commutative diagram

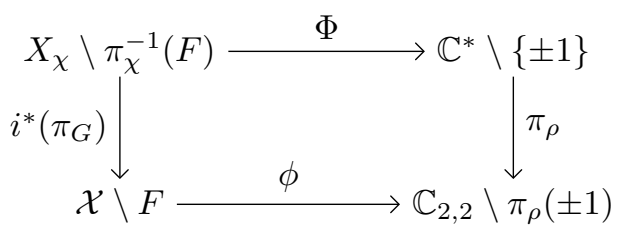

where $\pi_{\chi}$ (resp. $\pi_{\rho}$ ) is the projection $X_{\chi} \rightarrow X$ (resp. $\mathbb{C}^{*} \rightarrow \mathbb{C}_{2,2}$ as above). This diagram induces the isomorphism

$$
\pi_{1}(\mathcal{X} \backslash F) / \pi_{1}\left(X_{\chi} \backslash \pi_{\chi}^{-1}(F)\right)=\pi_{1}\left(\mathbb{C}_{2,2} \backslash \pi_{\rho}( \pm 1)\right) / \pi_{1}\left(\mathbb{C}^{*} \backslash\{ \pm 1\}\right),
$$

of order 2 quotients, which shows that the pencils $\mathcal{X} \rightarrow \mathbb{C}_{2,2}$ preserve markings.

Finally, we will check that any such pencil $\Phi: X_{\chi} \rightarrow \mathbb{C}^{*}$ can be assumed to have connected fibers and hence the induced morphism $\Phi_{*}$ on cohomology is surjective, which implies that the $n$ pencils can be found to be strongly independent. Consider the induced orbifold pencil $\phi: \overline{\mathcal{X}} \rightarrow \mathbb{P}_{2,2}^{1}$ and its Stein factorization $\overline{\mathcal{X}} \stackrel{\phi}{\rightarrow} S \stackrel{\tilde{\sigma}^{\prime}}{\rightarrow} \mathbb{P}_{2,2}^{1}$. Since $\overline{\mathcal{X}}$ is a rational surface, one has $S=\mathbb{P}^{1}$ with an orbifold structure containing 
at least two orbifold points each locally a quotient by an order 2 automorphism. The double cover of $S$ ramified along these two orbifold points after removing the preimage of the point at infinity by $\tilde{\sigma}$ induces maps $X_{\chi} \stackrel{\Phi^{\prime}}{\rightarrow} \mathbb{C}^{*} \stackrel{\sigma}{\rightarrow} \mathbb{C}^{*}$ where $\Phi=\sigma \circ \Phi^{\prime}$ and $\Phi^{\prime}$ has connected fibers.

The moreover part is a direct consequence of Proposition 2.7.

\section{Pencils on the complements of plane curves and zero- dimensional components of characteristic varieties}

\subsection{Essential coordinate components and weight}

Essential coordinate components were defined and studied in [4]; the first appeared in [12]. The dimension of the essential coordinate component is zero since the pencil $f: \mathbb{C}^{2} \backslash D \rightarrow C$, corresponding to a positive-dimensional coordinate component of $V_{i}$, can be extended to a map $\bar{f}: \mathbb{C}^{2} \backslash D^{\prime} \rightarrow C$ with $D^{\prime} \subsetneq C$ (see [26]). It follows from [27] that essential coordinate characters have finite order. Now we can give the Hodge-theoretical characterization of such characters in terms of weights (see Definition 2.9) stated in Theorem 1.4.

Proof of Theorem 1.4. Let $\chi$ be an essential coordinate character and let $n$ be its order. Denote by $X_{n}$ the covering space of the complement to the curve corresponding to the surjection $\pi_{1}(X) \rightarrow H_{1}(X, \mathbb{Z} / n \mathbb{Z})$. Let $\bar{X}_{n}$ be a smooth model of the compactification of $X_{n}$. Then $W_{1} H^{1}\left(X_{n}, \mathbb{C}\right)=\operatorname{Im} H^{1}\left(\bar{X}_{n}, \mathbb{C}\right)$ (see [13]). If $v \in W_{1} H^{1}\left(X_{n}, \mathbb{C}\right)$ is a $\chi$-eigenvector of $H_{1}(X, \mathbb{Z} / n)$ then $\chi$ is the eigencharacter of the action on the cohomology of the branched cover. Since $\chi$ is a coordinate character, it follows from Sakuma's formula (see Theorem 2.15) that $\chi$ belongs to the characteristic variety of the curve $D^{\prime}$ with components corresponding to the nontrivial coordinates of $\chi$. Using Proposition 2.10, we obtain that essential coordinate characters have weight 2 .

Conversely, if a character has weight 2 , then it must be coordinate since by Theorem 2.15 noncoordinate characters belong to the image of $H^{1}\left(\bar{X}_{n}, \mathbb{C}\right) \rightarrow H^{1}\left(X_{n}, \mathbb{C}\right)$ and hence have weight one. This is essential since otherwise Theorem 2.15 would imply that it appears as the eigencharacter of a weight one subspace.

\subsection{2-torsion characters and quasitoric relations}

As a consequence of Theorem 1.3.(2) one has the following interpretation of depth for coordinate 2 -torsion characters in terms of quasitoric relations of type $(2,2,0)$ (see [11] for a detailed treatment of quasitoric relations of elliptic type).

Let $D \subset \mathbb{P}^{2}$ be a plane curve and let $\chi$ be a 2-torsion character of $\pi_{1}\left(\mathbb{P}^{2}-D\right)$ having 2 as the only weight (i.e., as in Theorem 1.4). Let $S$ be the collection of the irreducible divisors of $D$ and let $G(S)$ be the subgroup of the group of divisors of $\mathbb{P}^{2}$ generated by $S$.

We fix a generic line at infinity and identify the coordinate ring of the affine plane with $\mathbb{C}[x, y]$. An element in $\mathbb{C}(x, y)$ is an $S$-unit (see [30]) if it is the quotient 
of two polynomials such that the irreducible components of their zero locus belong to $S$. This is a multiplicative group denoted by $E(S)$. Note that one has the identification $E(S) / \mathbb{C}^{*} \cong G(S)$.

An $S$-unit is called primitive if it is a square-free polynomial. The set $S$ splits into two subsets $S=S_{0} \cup S_{1}$ depending on whether or not $\chi$ ramifies along each divisor, namely, $\chi\left(\gamma_{0}\right)=1$ (resp. $\chi\left(\gamma_{1}\right)=-1$ ) for a meridian $\gamma_{0}\left(\right.$ resp. $\gamma_{1}$ ) of a component of $S_{0}$ (resp. $S_{1}$ ). Let $D_{1}=\Sigma_{D_{1, i} \in S_{1}} D_{1, i}$ and $D_{0}=\Sigma_{D_{0, i} \in S_{0}} D_{0, i}$. Note that $D_{1}$ necessarily has even degree. In other words, $D$ admits an equation $F H=0$, where $(F)=D_{1}$ is a polynomial of even degree and $(H)=D_{0}$.

We shall use the following notations:

- $E(S)^{+}=E(S) \cap \mathbb{C}[x, y]$, i.e., $E(S)^{+}$is the multiplicative monoid generated by $\mathbb{C}^{*}$ and the polynomials in $E(S)$.

- $\mathbb{C}[x, y]_{E(S)}$ is the localization of $\mathbb{C}[x, y]$ at $E(S)^{+}$, the group of polynomial $S$-units. Hence $E(S)^{+}$is the group of units of $\mathbb{C}[x, y]_{E(S)}$.

- $\mathbb{K}_{F}:=\mathbb{C}(x, y)[\sqrt{F}]$ is the quadratic extension of $\mathbb{C}(x, y)$.

- $\mathbb{K}_{S}:=\mathbb{C}(x, y)[\sqrt{S}]$, the abelian extension of $\mathbb{C}(x, y)$ generated by $\sqrt{S}$, i.e., the set of multivalued functions whose square has an associated divisor which is in $G(S)$.

- $E(\sqrt{S})$ is the multiplicative group generated by $\mathbb{C}^{*}$ and $\sqrt{S}$ and $E(\sqrt{S})^{+}$is its associated monoid.

- $E_{P}(\sqrt{S})^{+}$is the set of primitive elements of $E(\sqrt{S})$, that is, $E_{P}(\sqrt{S})^{+}:=$ $\left\{\alpha \in E(\sqrt{S}) \mid \alpha^{2} \in E(S)^{+}\right.$is square-free $\}$.

We have the following inclusions:

$$
\begin{aligned}
& \mathbb{C}[x, y] \subset \mathbb{C}[x, y]_{E(S)} \subset \mathbb{C}(x, y) \subset \mathbb{K}_{F} \subset \mathbb{K}_{S} \\
& E(S)^{+} \subset E(S) \quad E(\sqrt{S}) \supset E(\sqrt{S})^{+} \supset E_{P}(\sqrt{S})^{+} .
\end{aligned}
$$

We consider the set

$$
G_{S}:=\left\{(\bar{u}, \bar{v}) \in\left(\mathbb{K}_{S}\right)^{2} \mid \bar{u}^{2}-\bar{v}^{2}=1\right\}
$$

with the group structure given by

$$
\left(\bar{u}_{1}, \bar{v}_{1}\right) \cdot\left(\bar{u}_{2}, \bar{v}_{2}\right):=\left(\bar{u}_{1} \bar{u}_{2}+\bar{v}_{1} \bar{v}_{2}, \bar{u}_{1} \bar{v}_{2}+\bar{v}_{1} \bar{u}_{2}\right) .
$$

Note that $G_{S}$ is isomorphic to $\mathbb{K}_{S}^{*}$ via the map

$$
\begin{aligned}
\pi: \mathbb{K}_{S}^{*} & \rightarrow G_{S} \\
t & \mapsto\left(\frac{t+t^{-1}}{2}, \frac{t-t^{-1}}{2}\right) .
\end{aligned}
$$

Also, the map $\pi$ is equivariant with respect to the automorphisms $t \mapsto t^{-1}$ of $\mathbb{K}_{S}^{*}$ and $(u, v) \mapsto(u,-v)$ of $G_{S}$. 
Definition 5.1. Let $(\bar{u}, \bar{v}) \in G_{S}$. We say that $(\bar{u}, \bar{v})$ is an F-pair if

(1) $\bar{u}, \bar{v} \in E(\sqrt{S}) \cdot \mathbb{C}[x, y]_{E(S)}$ and

(2) there exists a decomposition $\bar{u}=\alpha \cdot u, \bar{v}=\beta \cdot v$, for some $\alpha, \beta \in E(\sqrt{S})$ and $u, v \in \mathbb{C}[x, y]_{E(S)}$, such that $\alpha \cdot \beta \in \sqrt{F} \cdot E(S)$.

A decomposition satisfying (2) is called an $F$-decomposition of the $F$-pair.

Remark 5.2. Note that any decomposition $\bar{u}=\alpha \cdot u, \bar{v}=\beta \cdot v$, for some $\alpha, \beta \in$ $E(\sqrt{S})$ and $u, v \in \mathbb{C}[x, y]_{E(S)}$ of an $F$-pair is an $F$-decomposition.

Lemma 5.3. The set $G$ of $F$-pairs is a subgroup of $G_{S}$.

Proof. Since $(\bar{u}, \bar{v})^{-1}=(\bar{u},-\bar{v})$ it is enough to prove that the condition of being an $F$-pair is preserved by the product. Consider $\left(\bar{u}_{1}, \bar{v}_{1}\right),\left(\bar{u}_{2}, \bar{v}_{2}\right) \in G$ and let $\bar{u}_{i}=\alpha_{i} \cdot u_{i}, \bar{v}_{i}=\beta_{i} \cdot v_{i}, i=1,2$, be $F$-decompositions of these pairs. Then

$$
\left(\bar{u}_{1}, \bar{v}_{1}\right) \cdot\left(\bar{u}_{2}, \bar{v}_{2}\right)=\left(\alpha_{1} \alpha_{2} u_{1} u_{2}+\beta_{1} \beta_{2} v_{1} v_{2}, \alpha_{1} \beta_{2} u_{1} v_{2}+\beta_{1} \alpha_{2} v_{1} u_{2}\right) .
$$

For the first coordinate we have

$$
\alpha_{1} \alpha_{2} u_{1} u_{2}+\beta_{1} \beta_{2} v_{1} v_{2}=\alpha_{1} \alpha_{2}\left(u_{1} u_{2}+\left(\alpha_{1} \beta_{1}\right)\left(\alpha_{2} \beta_{2}\right) \frac{v_{1} v_{2}}{\alpha_{1}^{2} \alpha_{2}^{2}}\right) .
$$

Note that $\alpha_{i}^{2}, \alpha_{i} \cdot \beta_{i} \in E(S), i=1,2$, and hence we have a decomposition of this first coordinate. In a similar way we obtain a decomposition of the second coordinate where the first factor is $\alpha_{1} \beta_{2}$. Since

$$
\left(\alpha_{1} \alpha_{2}\right)\left(\alpha_{1} \beta_{2}\right)=\alpha_{1}^{2}\left(\alpha_{2} \beta_{2}\right) \in \sqrt{F} \cdot E(S)
$$

the result follows.

Definition 5.4. Let $(\bar{u}, \bar{v}) \in G$ be an $F$-pair and let $\bar{u}=\alpha \cdot u, \bar{v}=\beta \cdot v$, be an $F$-decomposition of $(\bar{u}, \bar{v})$. This decomposition is said to be normal if

(1) $\alpha=\tilde{\alpha} / \gamma, \beta=\tilde{\beta} / \gamma, \quad$ where $\tilde{\alpha}, \tilde{\beta}, \gamma \in E_{P}(\sqrt{S})^{+}, \quad \operatorname{gcd}(\tilde{\alpha}, \tilde{\beta}, \gamma)=1$;

(2) $u=\tilde{u} / w, v=\tilde{v} / w ; \quad \tilde{u}, \tilde{v} \in \mathbb{C}[x, y], w \in E(S)^{+}, \quad \operatorname{gcd}(\tilde{u}, \tilde{v}, w)=1 ;$

(3) $(\tilde{\alpha} \tilde{\beta})^{2}=F$;

(4) $\gamma^{2}$ is a divisor of $H$.

Remark 5.5. The group $\mathbb{C}^{*}$ acts on the set of $F$-normal decompositions by

$$
\lambda \cdot(\alpha \cdot u, \beta \cdot v)\left((\lambda \alpha) \cdot\left(\lambda^{-1} u\right),\left(\lambda^{-1} \beta\right) \cdot(\lambda v)\right)
$$

This action of $\mathbb{C}^{*}$ will be referred to as proportionality.

The following result shows that a normal $F$-decomposition of an $F$-pair is almost determined by the pair.

Proposition 5.6. Any F-pair admits a normal F-decomposition. Moreover, such an F-decomposition is unique (up to proportionality). 
Proof. The uniqueness part is straightforward. We start with an arbitrary $F$ decomposition $\bar{u}=\alpha \cdot u, \bar{v}=\beta \cdot v$. We can for instance write $\alpha=\tilde{\alpha} / \gamma$, where $\tilde{\alpha}, \gamma \in E(\sqrt{S})^{+}$have no common factors. Note that an element in $E(\sqrt{S})^{+}$is not primitive if an only if it contains a factor in $E(S)^{+}$. If $\tilde{\alpha}$ is not primitive, then $\tilde{\alpha}=\tilde{\alpha}_{1} u_{1}$, where $u_{1} \in E(S)^{+}$. In this case, one can rewrite $\bar{u}=\left(\tilde{\alpha}_{1} / \tilde{\gamma}\right)\left(u_{1} u\right)$. One can assume that both decompositions $\bar{u}=\alpha \cdot u$ and $\bar{v}=\beta \cdot v$ are such that $\alpha$ and $\beta$ have primitive numerators and denominators. Then, after taking a common denominator for $\alpha$ and $\beta$ (resp. for $u$ and $v$ ), we can assume that the decomposition

$$
\begin{aligned}
& \bar{u}=\alpha \cdot u=\left(\frac{\tilde{\alpha}}{\gamma}\right) \cdot\left(\frac{\tilde{u}}{w}\right), \\
& \bar{v}=\beta \cdot v=\left(\frac{\tilde{\beta}}{\gamma}\right) \cdot\left(\frac{\tilde{u}}{w}\right)
\end{aligned}
$$

satisfies $5.4(1)$ and $5.4(2)$.

By Remark 5.2, the decomposition $(\alpha \cdot u, \beta \cdot v)$ is an $F$-decomposition and hence $\alpha \beta=\tilde{\alpha} \tilde{\beta} / \gamma^{2} \in \sqrt{F} \cdot E(S)$. Let $\sigma \in E_{P}(\sqrt{S})$ denote an irreducible element. We denote by $m(\sigma, \alpha)$ the multiplicity of $\sigma$ in the decomposition of $\alpha$ into irreducible factors. Since $\tilde{\alpha}, \tilde{\beta}, \gamma \in E_{P}(\sqrt{S})^{+}$one concludes that $m(\sigma, \bullet) \in$ $\{0,1\}$ for $\bullet=\tilde{\alpha}, \tilde{\beta}, \gamma$. Moreover, by condition (2) in Definition 5.1, if $\sigma^{2} \mid F$, then $m(\sigma, \tilde{\alpha})+m(\sigma, \tilde{\beta})-2 m(\sigma, \gamma)$ is odd and hence so is $m(\sigma, \tilde{\alpha})+m(\sigma, \tilde{\beta})$.

The previous two conditions imply that

- $m(\sigma, \tilde{\alpha})+m(\sigma, \tilde{\beta})-2 m(\sigma, \gamma)=1$,

- $m(\sigma, \gamma)=0$, and thus

- $m(\sigma, \tilde{\alpha})+m(\sigma, \tilde{\beta})=1$.

Hence the second property implies condition (4).

Similarly, if $\sigma^{2} \mid H$, then condition (2) in Definition 5.1, implies that $m(\sigma, \tilde{\alpha})+$ $m(\sigma, \tilde{\beta})-2 m(\sigma, \gamma)$ is even and hence so is $m(\sigma, \tilde{\alpha})+m(\sigma, \tilde{\beta})$. As above, this and the fact that $m(\sigma, \bullet) \in\{0,1\}$ for $\bullet=\tilde{\alpha}, \tilde{\beta}, \gamma$ imply that

- $m(\sigma, \tilde{\alpha})=m(\sigma, \tilde{\beta})=0$ and $m(\sigma, \gamma) \in\{0,1\}$, or

- $m(\sigma, \tilde{\alpha})=m(\sigma, \tilde{\beta})=1$ and $m(\sigma, \gamma)=0$.

In order to show condition (3) it is enough to prove that the last case can be avoided. In order to do so we rewrite $\bar{u}$ and $\bar{v}$ as

$$
\begin{aligned}
& \bar{u}=\left(\frac{\tilde{\alpha} / \sigma}{\sigma \gamma}\right) \cdot\left(\frac{\sigma^{2} \tilde{u}}{w}\right)=\alpha_{1} \cdot u_{1}, \\
& \bar{v}=\left(\frac{\tilde{\beta} / \sigma}{\sigma \gamma}\right) \cdot\left(\frac{\sigma^{2} \tilde{v}}{w}\right)=\beta_{1} \cdot v_{1},
\end{aligned}
$$

where $\alpha_{1}:=\tilde{\alpha}_{1} / \gamma_{1}, \beta_{1}:=\tilde{\beta}_{1} / \gamma_{1}, \tilde{\alpha}_{1}:=\tilde{\alpha} / \sigma, \tilde{\beta}_{1}:=\tilde{\beta} / \sigma, \gamma_{1}:=(\sigma \gamma), u_{1}:=\tilde{u}_{1} / w$, $v_{1}:=\tilde{v}_{1} / w, \tilde{u}_{1}:=\sigma^{2} \tilde{u}$, and $\tilde{v}_{1}:=\sigma^{2} \tilde{v}$. Therefore, the second case can be avoided. After a finite number of steps one can assume that $\bar{u}=\alpha_{1} \cdot u_{1}, \bar{v}=\beta_{1} \cdot v_{1}$ satisfies

1. $m\left(\sigma, \tilde{\alpha}_{1}\right)+m\left(\sigma, \tilde{\beta}_{1}\right)=1$ for all $\sigma$ such that $\sigma^{2} \mid F$,

2. $m\left(\sigma, \tilde{\alpha}_{1}\right)=m\left(\sigma, \tilde{\beta}_{1}\right)=0$ and $m\left(\sigma, \gamma_{1}\right) \in\{0,1\}$, for all $\sigma$ such that $\sigma^{2} \mid H$. 
Hence it satisfies (3). Finally, based on the construction, it is easy to check that this new decomposition also satisfies (1), (2), and (4), that is,

1. $\tilde{\alpha}_{1}, \tilde{\beta}_{1} \in E_{P}(\sqrt{S})^{+}, \operatorname{gcd}\left(\tilde{\alpha}_{1}, \tilde{\beta}_{1}, \gamma_{1}\right)=1$,

2. $\tilde{u}_{1}, \tilde{v}_{1} \in \mathbb{C}[x, y], w \in E(S)^{+}, \operatorname{gcd}\left(\tilde{u}_{1}, \tilde{v}_{1}, w\right)=1$, and

4. $\gamma_{1}^{2}$ is a divisor of $H$.

Consider a quintuple $(f, g, h, U, V)$ of polynomials in $\mathbb{C}[x, y]$ satisfying the functional equation

$$
f U^{2}-g V^{2}=h
$$

where $f \cdot g=F, h_{\text {red }}$ (a generator of the radical of $(h)$ ) divides $H$ and $F$, and $H$ are defined as at the beginning of this section. Note that $\left(\mathbb{C}^{*}\right)^{2}$ acts on the set of such quintuples as follows: given $\lambda, \mu \in \mathbb{C}^{*}$, then

$$
(\tilde{f}, \tilde{g}, \tilde{h}, \tilde{U}, \tilde{V})=\left(\left(\lambda^{2} f\right),\left(\lambda^{-2} g\right),\left(\mu^{2} h\right),\left(\mu \lambda^{-1} U\right),(\mu \lambda V)\right) .
$$

As in the case of $F$-pairs we will refer to such an action as proportionality.

Definition 5.7. A $(2,2,0)$-quasitoric relation associated with the character $\chi$ is a proportionality class (in the above sense) of quintuples $(f, g, h, U, V)$ of polynomials in $\mathbb{C}[x, y]$ satisfying the functional equation $f U^{2}-g V^{2}=h$, where $f \cdot g=F, h_{\text {red }}$ divides $H$ and $F$ and $H$ are defined as at the beginning of this section.

Remark 5.8. As mentioned before Definition 5.1, the set of $(2,2,0)$-quasitoric relations has a natural order two action defined by $(f, g, h, U, V) \mapsto(f, g, h, U,-V)$. Note that $(f, g, h,-U, V)$ is proportional to $(f, g, h, U,-V)$ and $(f, g, h,-U,-V)$ is proportional to $(f, g, h, U, V)$.

Our purpose now is to establish an isomorphism between $(2,2,0)$-quasitoric relations and normal $F$-decompositions. Recall the group $G$ of $F$-pairs from Lemma 5.3. Denote by $Q_{(D, \chi)}$ the set of $(2,2,0)$-quasitoric relations of $D$ associated with the character $\chi$, that is,

$Q_{(D, \chi)}:=\left\{(f, g, h, U, V) \in \mathbb{C}[x, y]^{5} \mid f U^{2}-g V^{2}=h, f \cdot g=F\right.$, and $\left.h_{\text {red }} \mid H\right\} / \sim$.

Proposition 5.9. The set of $(2,2,0)$-quasitoric relations $Q_{(D, \chi)}$ has a group structure isomorphic to the group $G$ of F-pairs, where the isomorphism is equivariant with respect to the order 2 actions on both groups.

Proof. Let $(\bar{u}, \bar{v})$ be an $F$-pair. We are going to associate with $(\bar{u}, \bar{v})$ a $(2,2,0)$ quasitoric relation $Q T(\bar{u}, \bar{v})$. To this end, consider a normal $F$-decomposition, $u=\alpha \frac{U}{W}, v=\beta \frac{V}{W}$, where $\alpha:=\tilde{\alpha} / \gamma$ and $\beta:=\tilde{\beta} / \gamma$. Let $f:=\tilde{\alpha}^{2}, g:=\tilde{\beta}^{2}, h_{0}:=\gamma^{2}$. From $\bar{u}^{2}-\bar{v}^{2}=1$, we deduce

$$
f U^{2}-g V^{2}=h_{0} W^{2} .
$$


It is enough to show that $W_{\text {red }}$ divides $H$. We already know that it divides $F H$, hence it is enough to show that no irreducible component of $F$ divides $W$. Otherwise it must divide either $f$ or $g$, say $f$ for simplicity, and then it divides $V$ (and not $g$ ). Therefore its multiplicity is odd in $f U^{2}$ and even in $g V^{2}$ as well as in $h_{0} W^{2}$, which is a contradiction. If $h:=h_{0} W^{2}$, then $Q T(\bar{u}, \bar{v}):=(f, g, h, U, V)$ is a $(2,2,0)$-quasitoric relation of $\chi$.

Notice that up to proportionality, one has $u=(\lambda \alpha) \frac{\lambda^{-1} \mu U}{\mu W}$ and $v=\left(\lambda^{-1} \beta\right) \frac{\lambda \mu V}{\mu W}$, where $\lambda \alpha:=\lambda \tilde{\alpha} / \gamma$ and $\lambda^{-1} \beta:=\lambda^{-1} \tilde{\beta} / \gamma$. Define $\tilde{f}:=\left(\lambda^{2} f\right), \tilde{g}:=\left(\lambda^{-2} g\right)$, $\tilde{W}:=\left(\mu^{2} W^{2}\right), \tilde{U}:=\left(\mu \lambda^{-1} U\right)$, and $\tilde{V}:=(\mu \lambda V)$. From $\bar{u}^{2}-\bar{v}^{2}=1$, we deduce

$$
\tilde{f} \tilde{U}^{2}-\tilde{g} \tilde{V}^{2}=h_{0} \tilde{W}^{2},
$$

and hence $\left(\tilde{f}, \tilde{g}, h_{0} \tilde{W}^{2}, \tilde{U}, \tilde{V}\right) \sim(f, g, h, U, V)$.

Conversely, fix a $(2,2,0)$-quasitoric relation $Q T=(f, g, h, U, V)$. Let $\tilde{\alpha}:=\sqrt{f}$ and $\tilde{\beta}:=\sqrt{g}$ and write $h:=h_{0} W^{2}$ where $h_{0}$ is square-free. Let $\gamma:=\sqrt{h_{0}}$. Then

$$
\left(\frac{\tilde{\alpha}}{\gamma} \frac{U}{W}, \frac{\tilde{\beta}}{\gamma} \frac{V}{W}\right)
$$

is the normal $F$-decomposition of the $F$-pair $(u, v)$ such that $Q T(u, v)=Q T$.

Finally, note that if $(\bar{u}, \bar{v})$ is the normal $F$-decomposition associated with the $(2,2,0)$-quasitoric relation $(f, g, h, U, V)$ as at the beginning of this proof, then $(\bar{u},-\bar{v})$ will be associated with $(f, g, h, U,-V)$, and hence the result follows.

Consider the double cover $X_{2}$ of $X=\mathbb{C}^{2} \backslash D$ associated with the order 2 character $\chi$.

Proposition 5.10. The group $Q_{(D, \chi)}$ is isomorphic to $\operatorname{Mor}_{\mathbb{Z}_{2}}\left(X_{2}, \mathbb{C}^{*}\right)$.

Proof. We will give a constructive proof of this result. Suppose one has a quasitoric relation $(f, g, h, U, V) \in Q_{(D, \chi)}$. There is a rational map $\mathbb{C}^{2} \rightarrow \mathbb{P}^{1}$ defined by $(x, y) \mapsto\left[f U^{2}: g V^{2}\right]$ such that $f U^{2}-g V^{2}=h$. This map, restricted to $\tilde{X}=$ $\{f g h=0\}$ defines an orbifold pencil $\tilde{X} \rightarrow \mathbb{C}_{2,2}=\mathbb{P}_{(2,[0: 1]),(2,[1: 0])}^{1} \backslash\{[1: 1]\}$. Finally, since $\{f g=0\}=\{F=0\}$ and $\{h=0\} \subset\{H=0\}$ by definition, one can restrict this to a well-defined orbifold pencil $(X, \chi) \rightarrow\left(\mathbb{C}_{2,2}, \rho\right)$, which induces an equivariant morphism in $\operatorname{Mor}_{\mathbb{Z}_{2}}\left(X_{2}, \mathbb{C}^{*}\right)$.

Conversely, any equivariant morphism in $\operatorname{Mor}_{\mathbb{Z}_{2}}\left(X_{2}, \mathbb{C}^{*}\right)$ induces a morphism of marked orbifolds on the quotient $(X, \chi) \rightarrow\left(\mathbb{C}_{2,2}, \rho\right)$. Extending this to $\mathbb{P}^{2}$ one obtains a rational morphism

$$
\mathbb{P}^{2}-\rightarrow \mathbb{C}_{2,2}=\mathbb{P}_{(2,[0: 1]),(2,[1: 0])}^{1} \backslash\{[1: 1]\},
$$

which on a generic affine chart can be defined by $(x, y) \mapsto\left[f U^{2}: g V^{2}\right]$, where $\{f g=0\}$ corresponds to the ramified part of $D$ and $f U^{2}-g V^{2}=h$ with $\{\tilde{h}=$ $0\} \subset\{H=0\}$ corresponds to the unramified part of $D$. This quasitoric relation defines an element of $Q_{(D, \chi)}$ and the order 2 action corresponds with the covering transformations. 
Finally, we check that this bijection is in fact a homomorphism. Note that an element $p:=(f, g, h, U, V) \in Q_{(D, \chi)}$ produces the commutative diagram

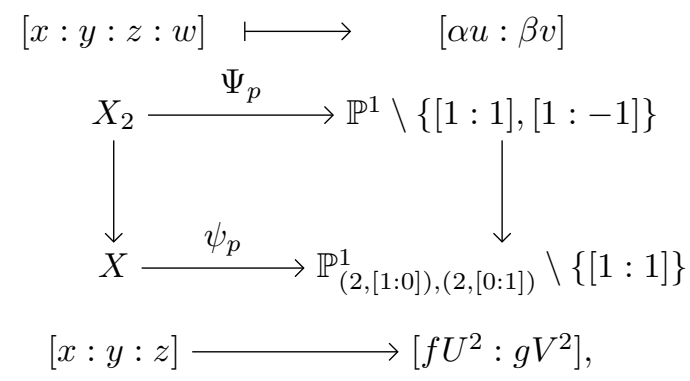

where $X_{2}$ is contained in $\left\{[x: y: z: w] \in \mathbb{P}^{3} \mid w^{2}=f g=F\right\}$ and $(\alpha u, \beta v)$ is the normal $F$-decomposition associated with $p$ according to Proposition 5.9. For convenience, we change the coordinates of $\mathbb{P}^{1}$ so that $[1: 1] \mapsto[1: 0]$ and $[1:-1] \mapsto$ $[0: 1]$. In this case, $\mathbb{P}^{1} \backslash\{[1: 1],[1:-1]\}$ becomes $\mathbb{C}^{*}=\mathbb{P}^{1} \backslash\{[1: 0],[0: 1]\}$ and the new equation of $\Psi_{p}: X_{2} \rightarrow \mathbb{C}^{*}$ becomes $\Psi_{p}(x, y, z, w)=(\alpha u+\beta v)^{2}$. Moreover, diagram (5.8) becomes

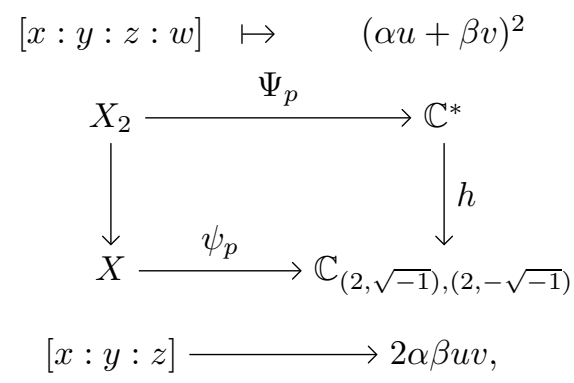

where the map $h$ is the double cover $t \mapsto-\left(t-t^{-1}\right) / 2$ ramified at $t= \pm \sqrt{-1}$ with values $\mp \sqrt{-1}$ and where $(\alpha u+\beta v)^{-1}=(\alpha u-\beta v)$.

Suppose given two quasitoric relations $p_{i}:=\left(f_{i}, g_{i}, h_{i}, U_{i}, V_{i}\right) \in Q_{(D, \chi)}, i=1,2$, where $f_{1} g_{1}=f_{2} g_{2}=F$ and $h_{i} \mid H$. Consider $\left(\bar{u}_{i}, \bar{v}_{i}\right)=\left(\alpha_{i} u_{i}, \beta_{i} v_{i}\right), i=1,2$, the normal $F$-decompositions associated with $p_{i}$ according to Proposition 5.9. From (5.1) one can see that

$$
\Psi_{p_{1} p_{2}}=\left(\bar{u}_{1} \bar{u}_{2}+\bar{v}_{1} \bar{v}_{2}+\bar{u}_{1} \bar{v}_{2}+\bar{u}_{2} \bar{v}_{1}\right)^{2}=\left(\bar{u}_{1}+\bar{v}_{1}\right)^{2}\left(\bar{u}_{2}+\bar{v}_{2}\right)^{2}=\Psi_{p_{1}} \Psi_{p_{2}} .
$$

As a consequence, under the conditions of Theorem 1.3 one obtains Theorem 1.5.

Proof of Theorem 1.5. The result follows from Proposition 5.10 and the fact that $\operatorname{Mor}_{\mathbb{Z}_{2}}\left(X_{2}, \mathbb{C}^{*}\right)=\operatorname{Hom}_{\mathbb{Z}_{2}}\left(\operatorname{Alb}\left(X_{2}\right)^{-}, \mathbb{C}^{*}\right)=\operatorname{Mor}_{\mathbb{Z}_{2}}\left(\left(\mathbb{C}^{*}\right)^{d}, \mathbb{C}^{*}\right)=\operatorname{Hom}\left(\mathbb{Z}^{d}, \mathbb{Z}\right)=\mathbb{Z}^{d}$, see (4.9), where $d \leq d(\chi)$ and equality always holds when the character has only weight 2 . 


\section{Examples}

We refer to [5] for explicit examples of characters of depth greater than one and corresponding orbifold pencils in the case of complements of reducible plane curves.

In this section we present two examples illustrating interesting phenomena about 2-torsion characters that have come up during the preparation of this paper and that might be of interest to the reader.

\subsection{Orbifold pencils of type $(2,2,2,2)$}

Note that Theorem 1.3 (2) refers only to weight 2 characters of order 2 . Characters of order 2 and weight 1 might be associated with elliptic orbifold pencils of type $(2,2,2,2)$ as the following example seems to suggest.

Consider the Hesse arrangement $\mathcal{H}$ of the twelve lines $\left\{\ell_{1}, \ldots, \ell_{12}\right\}$ joining the inflection points of a smooth plane cubic. It is easy to check that the system of cubics sharing the nine inflection points is a pencil with exactly four singular fibers. Each one of these fibers is a completely reducible curve given by three lines in general position. The four cubics $\mathcal{C}_{k}:=\left\{\ell_{3 k+1} \ell_{3 k+2} \ell_{3 k+3}=0\right\}, k=0,1,2,3$, belong to a pencil and their union gives the Hesse arrangement. After blowing up the base points of this pencil one obtains an elliptic fibration over $\mathbb{P}^{1}$ where $P_{k} \in \mathbb{P}^{1}$ is the image of the special fiber $\mathcal{C}_{k}, k=0,1,2,3$. If one further blows up one of the three double points in each special fiber, one obtains a rational surface $\tilde{\mathbb{P}}^{2}$, four exceptional vertical divisors $E_{0}, E_{1}, E_{2}, E_{3}$ (not sections), and twelve strict transforms $\tilde{\ell}_{i}, i=1, \ldots, 12$. The surface $X:=\tilde{\mathbb{P}}^{2} \backslash \cup_{i=1}^{12} \tilde{\ell}_{i}$ together with the elliptic fibration induces a well-defined orbifold pencil onto $\mathbb{P}_{\left(2, P_{0}\right),\left(2, P_{1}\right),\left(2, P_{2}\right),\left(2, P_{3}\right)}^{1}$ since the preimage of $P_{k}$ in $X$ is given by $2 E_{k}$ (in divisor notation).

\subsection{Ceva arrangements}

Note that the polynomials $f$ and $g$ in the group of quasitoric relations (5.7) must satisfy $f g=F$ but such a partition of $F$ might be different for different quasitoric relations as the following example shows. Consider the set of lines given in (CEVA). The curve $D:=\left\{\prod_{i=1}^{7} \ell_{i}=0\right\}$ is a realization of the special Ceva arrangement $\operatorname{CEVA}(2,1)$ (see Section 2.3.J, p. 81 in [10]) otherwise known as the non-Fano plane. In [5], a computation of the 2-torsion characters of $D$ is presented via orbifold pencils. In particular, consider $G:=\pi_{1}\left(\mathbb{P}^{2} \backslash D\right)$ (whose abelianization is $\left.\left(\mathbb{Z} \gamma_{1} \oplus \cdots \oplus \mathbb{Z} \gamma_{7}\right) /\left(\gamma_{1}+\cdots+\gamma_{7}\right)\right)$. A character of $G$ can be represented by a septuple of complex numbers whose product is 1 , the $i$-th coordinate representing the image of any meridian $\gamma_{i}$ around $\ell_{i}$. Hence, the element $\chi=(1,-1,-1,1,-1,-1,1)$ represents a character of $G$. In fact, it is well known that its depth is 2 . Note that $F=\ell_{2} \ell_{3} \ell_{5} \ell_{6}$ whereas $H=\ell_{1} \ell_{4} \ell_{7}$ according to the notation introduced in Section 5.2. Note that

$$
\begin{aligned}
& \ell_{2} \ell_{5}-\ell_{3} \ell_{6}=\ell_{1} \ell_{4} \\
& \ell_{2} \ell_{6}-\ell_{3} \ell_{5}=\ell_{4} \ell_{7}
\end{aligned}
$$


are quasitoric relations of type $(2,2,0)$ corresponding to the quintuples $q_{1}:=$ $\left(\ell_{2} \ell_{5}, \ell_{3} \ell_{6}, \ell_{1} \ell_{4}, 1,1\right)$ and $q_{2}:=\left(\ell_{2} \ell_{6}, \ell_{3} \ell_{5}, \ell_{4} \ell_{7}, 1,1\right)$ respectively. It is not hard to show that $q_{1}$ and $q_{2}$ are strongly independent and generate $\mathcal{Q}_{(D, \chi)}$. Note that $q_{1}$ and $q_{2}$ in fact determine two maps $\mathbb{P}^{2} \backslash C \rightarrow \mathbb{C} \backslash\{0,1\}$ and thus two surjections from $G$ onto $\pi_{1}(\mathbb{C} \backslash\{0,1\})=\mathbb{Z} * \mathbb{Z}$.

Finally, note that

$$
\ell_{2} \ell_{5} \ell_{8}^{2}-\ell_{3} \ell_{6} \ell_{9}^{2}=\ell_{1} \ell_{4} \ell_{7}^{2}
$$

where $\ell_{8}:=(y-z-x)$ and $\ell_{9}:=(z-x-y)$ is another quasitoric decomposition corresponding to $q_{3}=\left(\ell_{2} \ell_{5}, \ell_{3} \ell_{6}, \ell_{1} \ell_{4} \ell_{7}^{2}, \ell_{8}, \ell_{9}\right) \in \mathcal{Q}_{(D, \chi)}$. Following the formula (5.3) which describes explicitly the group structure in $\mathcal{Q}_{(D, \chi)}$ one can easily check that $q_{3}=-q_{1}+2 q_{2}$. Also note that $q_{3}$ determines an orbifold pencil $\mathbb{P}^{2} \backslash C \rightarrow \mathbb{C}_{2,2}$ and thus a surjection from $G$ onto the infinite dihedral group $\pi_{1}^{\text {orb }}\left(\mathbb{C}_{2,2}\right)=\mathbb{Z} / 2 * \mathbb{Z} / 2$ (also see $[8]$ ) which is a metacyclic group.

\section{References}

[1] Abel, N. H.: Sur l'intégration de la formule différentielle $\rho d x / \sqrt{R}, r$ et $\rho$ étant des fonctions entières. In Cuvres complètes de Niels Henrik Abel, Tome I. Imprimerie de Grøndahl \& Son, Christiania, 1981.

[2] Adem, A., Leida, J. And Ruan, Y.: Orbifolds and stringy topology. Cambridge Tracts in Mathematics 171, Cambridge University Press, Cambridge, 2007.

[3] Arapura, D.: Geometry of cohomology support loci for local systems. I. J. Algebraic Geom. 6 (1997), no. 3, 563-597.

[4] Artal, E., Carmona, J. and Cogolludo-Agustín, J. I.: Essential coordinate components of characteristic varieties. Math. Proc. Cambridge Philos. Soc. 136 (2004), no. 2, 287-299.

[5] Artal, E., Cogolludo-Agustín, J. I. And Libgober, A.: Depth of characters of curve complements and orbifold pencils. In Proceedings of the De Giorgi period in Pisa. Configuration Spaces: Geometry, Combinatorics, and Topology, 81-109. Publications of the Scuola Normale Superiore 14, Edizioni della Normale, Pisa, 2012.

[6] Artal, E., Cogolludo-Agustín, J. I. and Matei, D.: Orbifold groups, quasiprojectivity and covers. J. Singul. 5 (2012), 33-47. Proceedings of the International Conference on Singularity Theory and Applications Hefei, China, July 25-31, 2011.

[7] Artal, E., Cogolludo-Agustín, J. I. and Matei, D.: Characteristic varieties of quasi-projective manifolds and orbifolds. Geom. Topol. 17 (2013), no. 1, 273-309.

[8] Artal, E., Cogolludo-Agustín, J. I. and Tokunaga, H.: Pencils and infinite dihedral covers of $\mathbb{P}^{2}$. Proc. Amer. Math. Soc. 136 (2008), no. 1, 21-29.

[9] Artal, E., Cogolludo-Agustín, J. I. And Tokunaga, H.: A survey on Zariski pairs. In Algebraic geometry in East Asia, Hanoi 2005, 1-100. Adv. Stud. Pure Math. 50, Math. Soc. Japan, Tokyo, 2008.

[10] Barthel, G., Hirzebruch, F. And Höfer, T.: Geradenkonfigurationen und Algebraische Flächen. Aspects of Mathematics D4, Friedr. Vieweg \& Sohn, Braunschweig, 1987. 
[11] Cogolludo-Agustín, J. I. And Libgober, A.: Mordell-Weil groups of elliptic threefolds and the Alexander module of plane curves. To appear in J. Reine Angew. Math. DOI: 10.1515/crelle-2012-0096, January 2013.

[12] Cohen, D. C. And Suciu, A.I.: Characteristic varieties of arrangements. Math. Proc. Cambridge Philos. Soc. 127 (1999), no. 1, 33-53.

[13] Deligne, P.: Équations différentielles à points singuliers réguliers. Lecture Notes in Mathematics 163, Springer-Verlag, Berlin-New York, 1970.

[14] Deligne, P.: Théorie de Hodge. II. Inst. Hautes Études Sci. Publ. Math. (1971), no. $40,5-57$.

[15] Deligne, P.: Théorie de Hodge. III. Inst. Hautes Études Sci. Publ. Math. (1974), no. $44,5-77$.

[16] DimcA, A.: Pencils of plane curves and characteristic varieties. In Arrangements, local systems and singularities, 59-82. Progr. Math. 283, Birkhäuser Verlag, Basel, 2010 .

[17] Falk, M. And Yuzvinsky, S.: Multinets, resonance varieties, and pencils of plane curves. Compos. Math. 143 (2007), no. 4, 1069-1088.

[18] Friedman, R. And Morgan, J. W.: Smooth four-manifolds and complex surfaces. Ergebnisse der Mathematik und ihrer Grenzgebiete (3) 27, Springer-Verlag, Berlin, 1994.

[19] Hazama, F.: Pell equations for polynomials. Indag. Math. (N.S.) 8 (1997), no. 3, $387-397$.

[20] Hazama, F.: Twists and generalized Zolotarev polynomials. Pacific J. Math. 203 (2002), no. 2, 379-393.

[21] Hironaka, E.: Abelian coverings of the complex projective plane branched along configurations of real lines. Mem. Amer. Math. Soc. 105 (1993), no. 502, vi+85 pp.

[22] IitakA, S.: Logarithmic forms of algebraic varieties. J. Fac. Sci. Univ. Tokyo Sect. IA Math. 23 (1976), no. 3, 525-544.

[23] van Kampen, E. R.: On the fundamental group of an algebraic curve. Amer. $J$. Math. 55 (1933), no. 1-4, 255-260.

[24] Libgober, A.: Alexander invariants of plane algebraic curves. In Singularities, Part 2 (Arcata, Calif., 1981), 135-143. Proc. Sympos. Pure Math. 40, Amer. Math. Soc., Providence, RI, 1983.

[25] Libgober, A.: On the homology of finite abelian coverings. Topology Appl. 43 (1992), no. 2, 157-166.

[26] Libgober, A.: Characteristic varieties of algebraic curves. In Applications of algebraic geometry to coding theory, physics and computation (Eilat, 2001), 215-254. NATO Sci. Ser. II Math. Phys. Chem. 36, Kluwer Acad. Publ., Dordrecht, 2001.

[27] Libgober, A.: Non vanishing loci of Hodge numbers of local systems. Manuscripta Math. 128 (2009), no. 1, 1-31.

[28] Libgober, A.: On combinatorial invariance of the cohomology of the Milnor fiber of arrangements and the Catalan equation over function fields. In Arrangements of hyperplanes (Sapporo 2009), 175-187. Adv. Stud. Pure Math. 62, Math. Soc. Japan, Tokyo, 2012.

[29] MARCo, M. Á.: A description of the resonance variety of a line combinatorics via combinatorial pencils. Graphs Combin. 25 (2009), no. 4, 469-488. 
[30] Rosen, M.: $S$-units and $S$-class group in algebraic function fields. J. Algebra 26 (1973), 98-108.

[31] Sakuma, M.: Homology of abelian coverings of links and spatial graphs. Canad. J. Math. 47 (1995), no. 1, 201-224.

[32] Scott, P.: The geometries of 3-manifolds. Bull. London Math. Soc. 15 (1983), no. 5, 401-487.

[33] Serre, J. P.: Morphismes universels et variété d'Albanese. Séminaire Claude Chevalley 4 (1958-1959), 1-22.

[34] Shabat, G. And Zvonkin, A.: Plane trees and algebraic numbers. In Jerusalem combinatorics '93, 233-275. Contemp. Math. 178, Amer. Math. Soc., Providence, RI, 1994.

[35] Simpson, C.: A weight two phenomenon for the moduli of rank one local systems on open varieties. In From Hodge theory to integrability and TQFT $t t *$-geometry, 175-214. Proc. Sympos. Pure Math. 78, Amer. Math. Soc., Providence, RI, 2008.

[36] Suciu, A. I.: Translated tori in the characteristic varieties of complex hyperplane arrangements. Topology Appl. 118 (2002), no. 1-2, 209-223.

[37] Zariski, O.: On the irregularity of cyclic multiple planes. Ann. of Math. (2) 32 (1931), no. 3, 485-511.

[38] Zariski, O.: A theorem on the Poincaré group of an algebraic hypersurface. Ann. of Math. (2) 38 (1937), no. 1, 131-141.

Received April 27, 2012; revised October 1, 2013.

Enrique Artal Bartolo: Departamento de Matemáticas, IUMA, Universidad de Zaragoza, C. Pedro Cerbuna 12, 50009 Zaragoza, Spain.

E-mail: artal@unizar.es

José I. Cogolludo-Agustín: Departamento de Matemáticas, IUMA, Universidad de Zaragoza, C. Pedro Cerbuna 12, 50009 Zaragoza, Spain.

E-mail: jicogo@unizar.es

Anatoly Libgober: Department of Mathematics, University of Illinois, 851 S. Morgan Str., Chicago, IL 60607, USA.

E-mail: libgober@math.uic.edu

Partially supported by the Spanish Ministry of Education MTM2010-21740-C02-02. The third author was also partially supported by NSF and Simons Foundation grants while writing this paper. 